\title{
What is the relationship between photospheric flow fields and solar flares?
}

\author{
Brian T. Welsch \\ Space Sciences Laboratory, University of California, Berkeley, CA 94720-7450; \\ welsch@ssl.berkeley.edu \\ Yan Li \\ Space Sciences Laboratory, University of California, Berkeley, CA 94720-7450 \\ Peter W. Schuck \\ Heliophysics Science Division, Space Weather Laboratory, Code 674, NASA \\ Goddard Space Flight Center, 8801 Greenbelt Rd., Greenbelt, MD 20771 \\ George H. Fisher \\ Space Sciences Laboratory, University of California, Berkeley, CA 94720-7450
}

\begin{abstract}
We estimated photospheric velocities by separately applying the Fourier Local Correlation Tracking (FLCT) and Differential Affine Velocity Estimator (DAVE) methods to 2708 co-registered pairs of SOHO/MDI magnetograms, with nominal 96-minute cadence and $\sim 2^{\prime \prime}$ pixels, from 46 active regions (ARs) from 1996-1998 over the time interval $\tau_{45}$ when each AR was within $45^{\circ}$ of disk center. For each magnetogram pair, we computed the reprojected, average estimated radial magnetic field, $\tilde{B}_{R}$; and each tracking method produced an independently estimated flow field, u. We then quantitatively characterized these magnetic and flow fields by computing several extensive and intensive properties of each; extensive properties scale with AR size, while intensive properties do not depend directly on AR size. Intensive flow properties included moments of speeds, horizontal divergences, and radial curls; extensive flow properties included sums of these properties over each AR, and a crude proxy for the ideal Poynting flux, $S_{R}=\sum|\mathbf{u}| \tilde{B}_{R}^{2}$. Several quantities derived from $\tilde{B}_{R}$ were also computed, including: $\Phi$, total unsigned flux; $R$, a measure of the unsigned flux near strong-field polarity inversion lines (SPILs); and
\end{abstract}


$\sum \tilde{B}_{R}^{2}$. Next, using correlation and discriminant analysis, we investigated the associations between these properties and flares from the GOES flare catalog, when averaged over both $\tau_{45}$ and shorter time windows, of 6 and 24 hours. Our AR sample included both flaring and flare-quiet ARs; the latter did not flare above GOES C1.0 level during $\tau_{45}$. Among magnetic properties, we found $R$ to be most strongly associated with flare flux. Among extensive flow properties, the proxy Poynting flux, $S_{R}$, was most strongly associated with flare flux, at a level comparable to that of $R$. All intensive flow properties studied were more poorly associated with flare flux than these extensive properties. Past flare activity was also associated with future flare occurrence. The largest coefficients of determination from correlations with flare flux that we performed are $\sim 0.25$, implying no single variable that we considered can explain variations in average flare flux.

Subject headings: Sun: atmospheric motions; Sun: magnetic fields; Sun: flares

\section{Introduction}

Solar flares are intermittent releases of energy from the solar corona, in the form of accelerated electrons and ions, and strongly enhanced electromagnetic radiation over a wide range of wavelengths, from radio to X-ray. Consideration of the gravitational, thermal, and magnetic energy densities in the corona suggests that only the coronal magnetic field contains sufficient energy to account for the estimated energy release on flare time scales (Forbes 2000). Observations of stable, non-potential (e.g., twisted and/or sheared) magnetic field structures associated with coronal energy releases (e.g., sigmoids associated with coronal mass ejections [CMEs] studied by Canfield et al. 1999), have led to wide acceptance of the storage and release model of solar flares (see, e.g., Linker et al. 2001). In this model, magnetic free energy - energy above that of the potential magnetic field matching the same normal-field boundary condition at the base of the corona - is built up and stored in the corona on relatively long time scales (from several hours to weeks) and then suddenly released on much shorter time scales (minutes to a few hours). This naturally poses two questions: (1) What processes lead to the buildup of magnetic free energy in the corona? (2) And what processes lead to the sudden release of free magnetic energy in flares? 
Free magnetic energy in the corona can increase by: (a) flows that carry new flux from beneath the photosphere into the corona, and/or (b) photospheric flows that twist, shear, and/or cancel (Welsch 2006) already-emerged fields. Observations and theoretical models have demonstrated the viability of these mechanisms. Leka et al. (1996) convincingly demonstrated that twisted (non-potential) fields emerge through the photosphere, carrying free magnetic energy with them. Simulations of this emergence (e.g., Magara \& Longcope 2001; Fan 2001) produced sigmoidal (S- or inverse-S-shaped) field lines that may become visible in X-rays (e.g., Fan et al. 2003), and resemble the CME-associated sigmoids studied by Canfield et al. (1999). In already-emerged fields, Brown et al. (2003) found rotating sunspots in some eruptive active regions, and Welsch et al. (2004) observed shear flows along the eastern neutral line in NOAA AR 8210 prior to a flare and CME. In addition, rotating, shearing, and cancelling photospheric flows have been used to inject free magnetic energy in simulations (e.g., Lynch et al. 2008; Roussev et al. 2004; Amari et al. 2003; Lionello et al. 2002; Tokman \& Bellan 2002; Antiochos et al. 1999). While case studies of active region flows have been conducted (e.g., Keil et al. 1994; Li et al. 2004; Deng et al. 2006), investigations of the quantitative properties of photospheric flows and their relationships to flares in many active regions are lacking.

To remedy this shortcoming, we employed tracking techniques to estimate flows from magnetogram sequences of a few dozen active regions (ARs) that exhibited varying levels of flaring activity. We then investigated correlations between properties of the estimated flows and the occurrence of solar flares. We also studied relationships between flare occurrence and several properties of AR magnetic fields. Of particular note, two magnetic field properties that have been previously related to solar flares can serve as benchmarks for flow-flare associations: the total unsigned magnetic flux $\Phi$ of the AR at the photosphere (e.g., Leka \& Barnes 2007); and Schrijver's (2007) $R$ value, a measure of the unsigned flux near strong-field polarity inversion lines (SPILs).

As an aside, we note that Schrijver's $R$ value is very similar to a measure developed earlier by Falconer et al. (2003, 2006) - the length of SPILs, which should be strongly correlated with $R$ by virtue of the strong-field threshold. In fact, Barnes et al. (2009) report a correlation coefficient of 0.95 between these two quantities for their AR sample. Falconer et al. (2003, 2006) were among the first to quantitatively characterize the strong correlation between the presence of strongly non-potential photospheric magnetic fields and strong gradients in both radial and line-of-sight (LOS) magnetic fields. While the work Falconer et al. (2003, 2006) dealt with CMEs, and that of Schrijver (2007) dealt with flares, these two phenomena are clearly related (see, e.g., Andrews 2003). As a practical matter, we found Schrijver's measure 
simpler to compute than that of Falconer et al. (2003, 2006).

We are aware of studies of helicity evolution in active regions which suggest that the flux of magnetic helicity across the photosphere might play a role in solar flares (see, e.g., LaBonte et al. 2007). Tests of flow estimation methods using simulated data (Welsch et al. 2007), however, demonstrated that helicity estimates are often error-prone. Accordingly, we plan to investigate flare-helicity relationships in a future study, in which possible sources of error can be carefully examined.

In the next section, we describe our data set of magnetogram sequences from a few dozen ARs, and in $\S 3$, we describe how we estimated velocities from these sequences. In $\S 4$, we describe how we quantitatively characterized the intensive and extensive properties of the magnetic fields and estimated flows. In $\S 5$, we explore associations of these magnetic and flow properties with flares. Finally, in $\S 6$, we discuss what we have learned from our study.

\section{Data}

We chose to estimate flows from magnetic evolution observed in sequences of MDI (Scherrer et al. 1995) full-disk ( $\sim 2^{\prime \prime}$ pixels), 96-minute nominal cadence, LOS magnetograms. Compared to available magnetogram sequences with higher resolution and higher cadence (including some in which the full magnetic vector is estimated), the near-ubiquitous coverage of these full-disk LOS magnetograms is a tremendous advantage for tracking. After we began this study, the SOI/MDI team recalibrated field strengths in the MDI full-disk, 96-minute database; as our analysis had already progressed substantially, we chose to stick with the Level 1.8.0 data, which only included the plate scale correction. ${ }^{1}$

While sequences of white-light data could have been tracked instead, it is unclear precisely how white-light evolution is related to the underlying flow field. Further, evolution of the photospheric magnetic field is presumably more directly related to evolution of the coronal magnetic field - which powers flares - than evolution of photospheric white-light intensity. In addition, the relatively short lifetime of whitelight intensity features - most notably granules - places stringent limits on data cadence. Since magnetic structures appear much more persistent than white-light features, tracking with slower cadences is possible (though not necessarily desirable,

\footnotetext{
${ }^{1}$ See http://soi.stanford.edu/magnetic/Lev1.8/
} 
a point discussed further below).

We selected several dozen ARs and AR remnants from MDI synoptic maps from 1996 to 1998, from solar minimum into the ascending phase of the most recent cycle. Our sample was unbiased with respect to flare and CME productivity, but not with respect to AR morphology: we chose ARs that were, subjectively, both roughly bipolar and isolated (at the photosphere). Some of the AR remnants that we selected and tracked lacked sunspots, and therefore also lacked a NOAA AR designation; these have been excluded from the results presented here. The AR sample we use here is comprised of $N_{A R}=46$ ARs.

Properties of our AR data set are presented in Table 1. We only tracked each AR while it was within about $45^{\circ}$ of disk center as it transited the disk; the start and stop times for tracked intervals are shown in the table. To present a quantitative measure of the variability of flare activity within our sample, we have also included a measure of each AR's average daily GOES flux from flares at or above the C1.0 level,

$$
\overline{\mathcal{F}}_{45}=\left(100 S^{(X)}+10 S^{(M)}+1.0 S^{(C)}\right) / \tau_{45},
$$

where $\tau_{45}$ is the time interval (in days) that the AR was tracked across the portion of the disk within $45^{\circ}$ of disk center, and $S^{(i)}$ is the sum of GOES flare significands in the $i$-th GOES class over $\tau_{45}$, after Abramenko (2005). The units of $\overline{\mathcal{F}}_{45}$ are $\mu \mathrm{W} \mathrm{m}{ }^{-2}$ day $^{-1}$. To associate flares with ARs in our sample, we used flare source designations from the NOAA flare catalog, ${ }^{2}$ although this catalog is known to contain biases (Wheatland 2001). Flares associated with our ARs, during the time interval that each AR was tracked, include: zero A-class flares, 119 B-class flares, $154 \mathrm{C}$ class flares, $15 \mathrm{M}$-class flares, and two X-class flares. While the true frequency of occurrence of A- and B-class flares should exceed that of C-class flares, based upon the distribution of flare peak fluxes (e.g., Crosby et al. 1993), weak flares are often not ascribed to any AR in the GOES catalog, and we have only included flares with a source AR in our study. Of our $46 \mathrm{ARs}$, only 24 flared above the GOES C1.0 level while within $45^{\circ}$ of disk center; of the remaining 22 , a further 8 were identified as the source region of at least one sub-C-class flare.

\footnotetext{
${ }^{2}$ http://www.ngdc.noaa.gov/stp/SOLAR/ftpsolarflares.html\#xray
} 


\section{Methods}

The MDI instrument measures the LOS field strength, $B_{\mathrm{LOS}}$, averaged over each pixel. To estimate the radial field, $B_{R}$, from the observed $B_{\mathrm{LOS}}$, cosine corrections were applied to the LOS field in each pixel, $B_{R}=B_{\mathrm{LOS}} / \cos (\gamma)$, where $\gamma$ is the angle from disk center to each pixel.

To compensate for foreshortening, triangulation was used to interpolate the $B_{R}$ data - regularly gridded in the plane of the sky, but irregularly gridded in spherical coordinates $(\theta, \phi)$ on the solar surface - onto points $(x, y)$ corresponding to a regularly gridded Mercator projection of the spherical surface. This projection was adopted because it is conformal (locally shape-preserving), which is necessary to ensure displacements measured by our tracking algorithm were not biased in direction. The price of conformality is distortion of scale, such that apparent displacements are exaggerated by a factor of the secant of apparent latitude; but this is easily corrected after tracking. Details of our deprojection procedure are discussed in Appendix A.

We separately used the Fourier Local Correlation Tracking (FLCT; Fisher \& Welsch 2008) and the Differential Affine Velocity Estimator (DAVE; Schuck 2006) codes to produce two estimates of flows for each pair of successive magnetograms in our sequences. Prior to tracking, each pair of magnetograms was cross-correlated and then co-registered to sub-pixel accuracy using cubic-convolution interpolation. For FLCT, pixels below a $20 \mathrm{G}$ threshold in $\left|\bar{B}_{R}\right|$, where $\bar{B}_{R}$ is the average magnetic field for each magnetogram pair, were not tracked, nor were pixels farther than $\gamma=$ $45^{\circ}$ from disk center. DAVE estimated flows for many pixels that fell outside of these thresholds, but a filter was applied to the raw DAVE results that zeroed out flows from pixels where FLCT did not estimate flows. Because the physical length corresponding to Mercator pixels shrinks toward the poles, all estimated flows were corrected for the distortion in magnitude introduced by the Mercator projection. Moving cropping windows were applied to the deprojected magnetograms to extract subimages containing the ARs as they crossed the disk.

While the magnetogram cadence was nominally $\Delta t=96$ minutes, data gaps did exist. Flows estimated from magnetogram pairs with $\Delta t>$ six hours apart were excluded from the analyses of flow properties described in $\S 4$. With $\Delta t=96$ minutes, pixels of $\Delta s \sim 1400 \mathrm{~km}$, and flows of $\sim 0.3 \mathrm{~km} \mathrm{~s}^{-1}$ (cf., Hathaway et al. 2002), displacements would be $\sim 1.2$ pixels. We expect that this cadence effectively averages away flows with characteristic time- and length-scales shorter than a couple of hours and a couple of megameters. This implies that the flows we measure ought to be long-lived and large-scale relative to granular flows typically tracked from sequences 
of intensity images in white-light (e.g., November \& Simon 1988) or G-band.

Démoulin \& Berger (2003) asserted that tracking methods applied to magnetogram sequences estimate an apparent flow $\mathbf{u}$, with

$$
\mathbf{u} \equiv \mathbf{v}_{h}-v_{r} \mathbf{B}_{h} / B_{r},
$$

and where $\mathbf{v}$ is the plasma velocity, $\hat{\mathbf{r}}$ is the normal to the magnetogram surface, and $h$ denotes components in the magnetogram surface. Here, we are using $B_{R}$ as an estimate of $B_{r}$, the true normal (radial) field. Substitution of equation (2) into the normal component of the magnetic induction equation produces a continuity-like equation for the normal component of of the magnetic field and the flux transport velocity u (Démoulin \& Berger 2003; Schuck 2005). Démoulin \& Berger (2003) pointed out that knowledge of $\mathbf{u}$ and $\mathbf{B}$ is sufficient to compute the fluxes of magnetic energy and helicity across the magnetogram surface. However, recent tests of tracking methods on MHD simulation data by Welsch et al. (2007) cast doubt on the accuracy of methods that exclusively track the normal component of the magnetic field. A followup investigation by Schuck (2008) using the same MHD simulation data suggest that some tracking methods estimate $\mathbf{v}_{h}$ much more accurately than $\mathbf{u}$ - they produce a biased estimate of the horizontal plasma velocity. This supports the view that line-of-sight tracking methods are insensitive to the vertical flows that drive flux emergence (Démoulin \& Pariat 2009). However, these methods may produce a reasonable estimate of the fluxes associated with footpoint twisting in mature active regions. Tests conducted by both Welsch et al. (2007) and Schuck (2008) suggest that velocity estimates that employ the full magnetic vector - not just the LOS data that we use here - would be more accurate. Currently, however, sequences of vector magnetograms are too rare for statistical studies relating magnetic evolution to flares.

For tracking each active region's data cube, both FLCT and the DAVE require selecting a spatial windowing parameter, $\sigma$. In principle, this choice can be made objectively, by selecting the value of $\sigma$ that maximizes agreement between the observed magnetic evolution $\Delta B_{R} / \Delta t$ between magnetogram pairs, and the expected relationship (Démoulin \& Berger 2003) between this evolution and the observed flow $\mathbf{u}$,

$$
\nabla_{h} \cdot\left(\mathbf{u} \bar{B}_{R}\right)=-\Delta B_{R} / \Delta t,
$$

where $\bar{B}_{R}$ is the average of $B_{R}$ from each magnetogram in the pair. (But LCT schemes are not formally consistent with this continuity-like equation; see Schuck 2005.) In practice, the cadence in our study was so low that equation (3) was relatively poorly obeyed (compared to tests with other data sets with higher cadence) for any choice 
of $\sigma$. Tests that quantified agreement of DAVE results with equation (3) using slopefitting, as well as linear (Pearson) and rank-order correlations, suggested that $\sigma$ in the range of $8-10$ pixels maximized consistency across these measures. Accordingly, $\sigma=9$ pixels was used with the DAVE, and $\sigma=8$ pixels was used with FLCT. We expect this windowing leads to further spatial smoothing of flows, averaging over flows with characteristic length scales less than several megameters. Following Welsch et al. (2004), positive- and negative-flux pixels were tracked separately with FLCT, with these separate estimates combined into all-polarity flow maps.

The FLCT code used here was slightly modified from that described by Fisher \& Welsch (2008). The modified version ${ }^{3}$ removes the mean intensity from the subimages prior to cross correlating them. A Gaussian spatial frequency filter was used in the FLCT runs, with a roll-off wavenumber in $x$ of $k_{x \text {,roll-off }}=0.25 \max \left(k_{x}\right)$, and similarly for $y$. This has been found to improve accuracy in tests with known shifts (Fisher \& Welsch 2008), and to ameliorate FLCT's tendency to underestimate speeds.

Our full data set consists of $N_{\text {flow }}=2708$ sets of flows estimated by both FLCT and DAVE. A typical FLCT flow field is shown in Figure 1. This example was taken from AR 8038, an active region which produced three flares and CMEs as it crossed the disk in May 1997 ( $\mathrm{Li}$ et al. 2009). The time shown on the plot corresponds to the end of the 96-minute interval over which the flows were estimated. DAVE flows from tracking the same data are shown in Figure 2. While there are broad regions of agreement between the directions of FLCT and DAVE flows, there are also some regions where the flows are in the opposite direction. Our focus here is not to compare FLCT and DAVE flows, but rather to test the sensitivity of flow-flare correlations (or lack thereof) to the method chosen to estimate flows. Nonetheless, in Figure 3, we show scatter plots relating the $x$ - and $y$-components of FLCT and DAVE flows from the same time interval, to illustrate how the flows compare in this case. We only plotted results for the set of pixels in which both methods estimated flows. On the same plot, we also show the linear (Pearson) and rank-order correlations between the flow estimates. Clearly, the methods' flow estimates are significantly correlated, but do not agree in detail. For the $\sim 16.5$ million pixels for which both FLCT and DAVE estimated velocities, the linear and rank-order correlations of the $x$-components of the flows were 0.67, while for the $y$-components, these correlations were 0.60 and 0.61 , respectively. Also, the linear and rank-order correlations between FLCT and DAVE speeds, when both are weighted by the unsigned field strengths, are higher still —

\footnotetext{
${ }^{3}$ available at http://solarmuri.ssl.berkeley.edu/ fisher/public/software/FLCT/C_VERSIONS/
} 
0.85 and 0.76 , respectively. This suggests the flows tend to agree more closely in strong field regions.

Comparing speeds shows a systematic difference. Over the same set of $\sim 16.5$ million pixels, FLCT's mean and median flow speeds were 0.069 and $0.062 \mathrm{~km} \mathrm{sec}^{-1}$, while for DAVE, mean and median flow speeds were 0.112 and $0.099 \mathrm{~km} \mathrm{sec}^{-1}$. In tests with synthetic magnetograms from ANMHD simulations, Welsch et al. (2007) found that an older version of FLCT than that used here underestimated speeds by about $\sim 30 \%$, which would suggest a DAVE/FLCT slope of $0.7^{-1} \sim 1.4$. Here, the DAVE/FLCT speed ratio is nearer 1.6.

These flow speeds are significantly less than flows estimated by other methods, e.g., supergranular flow speeds determined from center-to-limb Doppler variation by Hathaway et al. (2002). They are, however, only slightly slower than flows estimated by Chae et al. (2004) for 96-minute cadence data; our slower speeds might result from our larger spatial windowing, $\sim 17 "$ (see their Fig. 7).

\section{Quantifying Magnetic Field and Flow Properties}

\subsection{Typical Fields and Changes}

To familiarize the reader with our data set, we present some statistics regarding typical magnetic fields and field changes in the approximately 21 million pixels in which $\left|\bar{B}_{R}\right|$ exceeded $20 \mathrm{G}$ (of about 85 million total pixels in our data set). Over this set of pixels, the median and average cosine-corrected, unsigned, pixel-averaged field strengths $\left|\bar{B}_{R}\right|$ were $63 \mathrm{G}$ and $113 \mathrm{G}$, respectively. The median and average unsigned rates of change, $\left|\Delta B_{R}\right| / \Delta t$ were $3 \times 10^{-3} \mathrm{G} \mathrm{s}^{-1}$ and $4 \times 10^{-3} \mathrm{G} \mathrm{s}^{-1}$, respectively, which correspond to unsigned changes of $17 \mathrm{G}$ and $23 \mathrm{G}$, respectively, for the nominal $\Delta t=5760 \mathrm{~s}$ time interval between magnetograms. Perhaps unsurprisingly, this is roughly the quoted noise level for MDI magnetograms. ${ }^{4}$ The linear and rank-order correlation coefficients between unsigned $\left|\bar{B}_{R}\right|$ and $\left|\Delta B_{R} / \Delta t\right|$ were 0.36 and 0.41 , respectively - hence, absolute changes tend to be larger in stronger fields. The linear and rank-order correlation coefficients for $\left|\bar{B}_{R}\right|$ and $\left|\left(\Delta B_{R} / \Delta t\right) / B_{R}\right|$ were -0.30 and -0.39 , respecively, implying relative changes tend to be smaller in stronger fields. The rank-order correlation between signed $\bar{B}_{R}$ and $\Delta B_{R} / \Delta t$ was 0.05 , which amounts to a small but statistically significant bias: negative fields were slightly more likely to

${ }^{4}$ See http://soi.stanford.edu/magnetic/Lev1.8/ 
get more negative, and positive fields were slightly more likely to get more positive. This imbalance, however, cannot be sustained indefinitely. Our ARs all have NOAA designations (and so possess sunspots), and therefore contain relatively newly emerged flux; perhaps older flux in decaying active regions behaves differently.

\subsection{Field Properties}

In this and the following subsections, we describe aspects of magnetic and flow fields that we calculate to relate to flare activity. We distinguish between "extensive" properties, which depend directly on AR size (e.g., integrated quantities), and "intensive" properties, which do not depend directly on AR size (e.g., averaged quantities). This is in analogy to thermodynamics, in which extensive quantities scale with the system size (e.g., total thermal energy, or particle number), while intensive properties do not (e.g., temperature, or particle density). As a rule, quantities that would increase if an AR's length scales were doubled (all else held equal) are extensive, e.g., total unsigned flux; quantities that would not increase are intensive, e.g., average magnetic field strength. Although our characterization of $\Phi$ as extensive is based upon a hypothetical doubling of AR area, we note that Fisher et al. (1998) found that $\Phi$ is in fact strongly positively correlated with (and nearly a linear function of) AR area. While Fisher et al. (1998) used the term "extrinsic" for $\Phi$ and other integral field properties, we prefer extensive for its more direct analogy to thermodynamics.

While our analysis centers on properties of estimated flows, we also characterized properties of the time-averaged estimated radial magnetic field $\bar{B}_{R}$ corresponding to each pair of field estimates used for tracking, as well as properties of changes in the magnetic field that do not require tracking. These magnetic field and field evolution properties are described below, and then summarized in Table 2.

In the subsequent statistical analyses of magnetic field properties, it will often be appropriate to use magnetic field values scaled in a way to compensate for the latitude-dependent area distortion introduced by the Mercator projection. This rescaling is necessary because the physical area represented by Mercator-projected pixels shrinks toward the poles (the effect which causes Greenland and Antarctica to appear unrealistically large in Mercator projections of the Earth's surface); details are given in Appendix A. Without rescaling, field strengths in Mercator pixels that represent a smaller area on the Sun would be given equal weighting in our analyses. The apparent area $d a$ of a Mercator-projected pixel - equal to the MDI disk-center 
pixel area, $(\sim 1.4 \mathrm{Mm})^{2}$ — is related to Mercator-corrected pixel area $d \tilde{a}$ via

$$
d \tilde{a}(\tilde{\theta})=d a \cos ^{2} \tilde{\theta}
$$

where $\tilde{\theta}$ denotes the latitude of each pixel (cf., co-latitude $\theta$ often used in spherical coordinates). Pixel values used in calculations involving local magnetic properties (e.g., gradients, contours, and as a threshold for tracking) do not require Mercator correction. In contrast, pixel values in global quantities (e.g., sums, averages, and higher moments of magnetic quantities) must be weighted by $\cos ^{2} \tilde{\theta}$. Because we frequently refer to Mercator-corrected magnetic field values, we define

$$
\tilde{B}_{R} \equiv \bar{B}_{R} \cos ^{2} \tilde{\theta}
$$

and

$$
\tilde{B}_{R}^{2} \equiv \bar{B}_{R}^{2} \cos ^{2} \tilde{\theta}
$$

Leka \& Barnes (2007) demonstrated that the total unsigned flux was strongly associated with flaring, so we calculated this extensive quantity from each average estimated radial field,

$$
\Phi=\sum\left|\bar{B}_{R}\right| d \tilde{a}=\sum\left|\tilde{B}_{R}\right| d a
$$

where the sum runs over the cropping window.

For each magnetogram, we also computed the first four moments of the estimated radial magnetic field, $\tilde{B}_{R}$ : mean $\left\langle\tilde{B}_{R}\right\rangle$, variance $\sigma^{2}\left[\tilde{B}_{R}\right]$, skewness $\zeta\left[\tilde{B}_{R}\right]$, and kurtosis $\kappa\left[\tilde{B}_{R}\right]$, after Leka \& Barnes (2003). We denote this family of moments $\mathcal{M}\left[\tilde{B}_{R}\right]$,

$$
\mathcal{M}\left[\tilde{B}_{R}\right] \equiv \begin{cases}\left\langle\tilde{B}_{R}\right\rangle & \text { mean } \\ \sigma^{2}\left[\tilde{B}_{R}\right] & \text { variance } \\ \zeta\left[\tilde{B}_{R}\right] & \text { skewness } \\ \kappa\left[\tilde{B}_{R}\right] & \text { kurtosis }\end{cases}
$$

Since each of these moments does not, of necessity, scale with active region size, these descriptors of AR magnetic fields are intensive.

Because flare processes might be nonlinearly related to photospheric field strength (because, for instance, magnetic energy density is proportional to magnetic field squared), we also computed

$$
\sum \tilde{B}_{R}^{2}
$$

where, as above, the sum runs over all pixels in each average magnetogram. While $\tilde{B}_{R}^{2}$ has units of magnetic energy density, we do not ascribe any particular physical 
meaning to the sum of this quantity; it is included in our analysis to provide baseline comparisons for other quantities. (We also investigated sums of higher powers of $\tilde{B}_{R}$, but linear and rank-order correlations of summed $\tilde{B}_{R}^{2}$ with summed $\left|\tilde{B}_{R}\right|^{3}$ and $\tilde{B}_{R}^{4}$ are all above 0.9 , implying these variables contained little additional information.)

Schrijver (2007) correlated the total unsigned flux, $R$, near strong-field PILs (SPILs) with flaring, so we also computed $R$ for each magnetogram in our data set. This entails: (1) creating two separate positive and negative bitmaps, representing regions of strong average field $\left(\left|\bar{B}_{R}\right|>150 \mathrm{G}\right)$ in each polarity; (2) computing the product of dilated versions of these bitmaps, to find loci with strong, opposite polarity fields in close proximity; (3) computing a SPIL-weighting function, $W_{S P I L}$, by convolving the product bitmap with a normalized Gaussian of 15 Mm FWHM; (4) summing the unsigned flux in the SPIL-weighted magnetogram,

$$
R=\sum W_{S P I L}\left|\tilde{B}_{R}\right| .
$$

A few of these steps are illustrated explicitly in Welsch \& Li (2008). Some magnetograms in our study have $R=0$. While $R$ quantifies one aspect of the internal arrangement of flux within an AR - how much lies near strong-field polarity inversion lines - it also scales with active region size, albeit with the length $L$ of each AR, not area $A$. Accordingly, we also characterize $R$ as extensive.

In addition to $\Phi$ and $R$, we calculated the time derivatives $\dot{\Phi}$ and $\dot{R}$ between averaged magnetograms (each in $\left[\mathrm{Mx} \mathrm{sec}^{-1}\right]$ ), to investigate the correlation between changes in these quantities and flares. If the flux emergence rate per unit area, in $\mathrm{Mx} \mathrm{sec}-1 \mathrm{~cm}^{-2}$, were uniform over the Sun, then the nearly linear dependence of $\Phi$ with the area $A$ over which magnetic flux is present (Fisher et al. 1998) would imply a nearly linear relationship between $\dot{\Phi}$ and $\Phi$ - essentially, the larger an AR's $\Phi$ is, the larger the cross-section for emergence into that AR. In fact, Harvey \& Zwaan (1993) showed that emergence rates are enhanced over the background level in areas where flux has already emerged. Hence, we characterize $\dot{\Phi}$ as extensive, and expect $\dot{\Phi}$ and $\Phi$ to be correlated. To account for this, we also calculated intensive measures of the rates of change of $\Phi$ and $R$, the flux-normalized $\dot{\Phi} / \Phi$ and $\dot{R} / R$, to compensate for correlations between $\dot{\Phi}$ and $\Phi$, and $\dot{R}$ and $R$.

Wang (2006) reported changes in the center-of-flux positions of each polarity associated with five flares (X- or M- class) in $\delta$-spot regions. Accordingly, we also computed the center-of-flux (COF) positions (Mercator corrected) of each AR polarity in each average cropped magnetogram,

$$
\mathbf{x}_{ \pm}=\sum_{ \pm} \mathbf{x}\left|\tilde{B}_{R}\right| / \sum_{ \pm}\left|\tilde{B}_{R}\right|
$$


where the \pm sums run over pixels of the corresponding polarity. We then computed the mean polarity separation vector,

$$
\mathbf{d x}=\mathbf{x}_{-}-\mathbf{x}_{+}
$$

which points from $\mathbf{x}_{+}$to $\mathbf{x}_{-}$, and found the rate of change $(\dot{\mathbf{d} x})$ (in $\left[\mathrm{km} \mathrm{sec}^{-1}\right]$ ) of $\mathbf{d x}$ between pairs of average magnetograms. We then computed the "center-of-flux divergence,"

$$
D_{C O F}=(\dot{\mathbf{d x}}) \cdot \mathbf{d x} /|\mathbf{d x}| \text {, }
$$

and normalized the change by the initial dx, as well as the "center-of-flux shear,"

$$
S_{C O F}=|\hat{\mathbf{r}} \cdot((\mathbf{d} \mathbf{x}) \times \mathbf{d} \mathbf{x})| /|\mathbf{d} \mathbf{x}|,
$$

implied by these changes. The unit vector $\hat{\mathbf{r}}$ is normal to the projected magnetogram plane. Both $D_{C O F}$ and $S_{C O F}$ have units of velocity. A negative (resp., positive) $D_{C O F}$ corresponds to centers of flux growing closer (resp., farther) along the line separating them. Based upon flux emergence simulations (e.g., Abbett et al. 2000), one might expect $D_{C O F}>0$ with the emergence of new flux, and, conversely, $D_{C O F}<0$ with flux cancellation. If centers of flux have a tendency to move closer together as a result of flares, as Wang (2006) found, then flares should be anti-correlated with $D_{C O F}$. A non-zero $S_{C O F}$ corresponds to displacements of centers of flux in opposite directions perpendicular to $\mathbf{d x}$.

\subsection{Flow Properties}

Here, we describe how we quantified apects of our estimated flows; these properties are also summarized in Table 2. For each pair of magnetograms we track, we typically have two independent flow estimates, by FLCT and DAVE, for each of several thousand pixels. From these estimates, we computed several intensive measures of flow properties. As with $\tilde{B}_{R}$, we computed the moments of each flow field, $\mathcal{M}[u]$. Because photospheric flows in regions of stronger $\left|\bar{B}_{R}\right|$ are expected to impact coronal evolution more strongly than regions with weak $\left|\bar{B}_{R}\right|$, we also computed flux-weighted moments $\mathcal{M}\left[u|d \Phi| / \Phi_{u}\right]$. In this expression, $|d \Phi|$ represents the (Mercator-corrected) flux in each pixel, and $\Phi_{u}$ is the total unsigned flux only from those pixels for which each method (FLCT or DAVE) estimated flows. (Averaging over the entire 2D arrays for each velocity estimate - which contain many zero velocities [e.g., from weak-field regions] could bias the moments if, for instance, low-flux ARs have fractionally more weak-field pixels.) We also computed several related extensive quantities: summed 
speeds and field-weighted speeds, $\sum u$ and $\sum u\left|\bar{B}_{R}\right|$, respectively; and the sum of a kinetic-energy like quantity, $\sum u^{2}$, and its field-weighted version, $\sum u^{2}\left|\bar{B}_{R}\right|$, respectively. A grayscale map of $u^{2}\left|\tilde{B}_{R}\right|$ is shown in the upper-left panel of Figure 4.

We also computed the sum of a quantity that, dimensionally, has units of an energy flux,

$$
S_{R} \equiv \sum u \tilde{B}_{R}^{2}
$$

with $u$ in $\mathrm{cm} \mathrm{sec}^{-1}$ (our default in this paper is $\mathrm{km} \mathrm{sec}^{-1}$ ) and $\tilde{B}_{R}^{2}$ in $\mathrm{G}^{2}$, the summand has units ergs $\mathrm{cm}^{-2} \mathrm{sec}^{-1}$. Multiplying by MDI's disk-center pixel area $d a, \sim 2 \times 10^{16}$ $\mathrm{cm}^{2}$, then yields units of power, ergs $\mathrm{sec}^{-1}$. This sum represents an extensive measure of the transport of magnetic energy at the photosphere. The true radial Poynting flux $S_{r}$ across the photosphere and into the corona can be derived from an estimated flow field $\mathbf{u}$ and a vector magnetogram (Démoulin \& Berger 2003) via,

$$
\begin{aligned}
S_{r} & =(1 / 4 \pi) \sum\left[\left(\mathbf{B}_{h} \cdot \mathbf{B}_{h}\right) v_{r}-\left(\mathbf{v}_{h} \cdot \mathbf{B}_{h}\right) B_{r}\right] \\
& =-(1 / 4 \pi) \sum\left(\mathbf{u} \cdot \mathbf{B}_{h}\right) B_{r}
\end{aligned}
$$

which depends sensitively on the relative orientation of the flow and magnetic fields. Our simple proxy $S_{R}$ completely ignores this dependence. As long as magnetic fields are not exactly vertical, and observations reported by Fisher et al. (1998) suggest they tend not to be, then strong horizontal fields will tend to occur where there are strong vertical fields. Further, flux emergence and submergence occurs on PILs; but our proxy is small along PILs, where the radial field vanishes. But flux from emerging (submerging) features should diverge (converge), which can be detected (see $\S 4.4$, below). Consequently, our proxy, crude though it may be, could be statistically related to actual energy fluxes. A grayscale map of $S_{R}$ is shown in the upper-right panel of Figure 4.

To characterize flow properties arising from the vector nature of our estimated flows, we computed several additional intensive properties of the flow. For each flow field $\mathbf{u}$, we computed the absolute and signed horizontal divergence, and the unsigned radial component of the vorticity (curl), and then computed moments of these distributions. As above, horizontal refers to directions perpendicular to the normal (radial) direction, $\hat{\mathbf{r}}$. In addition, we computed the flux-weighted signed and unsigned horizontal divergences, unsigned radial curls, and moments of these distributions. We excluded derivatives in pixels with any of the four-nearest neighbor pixels below the absolute field-strength tracking threshold, to avoid contributions to divergences and curls from flow discontinuities between tracked and untracked pixels. Weighted divergence and curl maps are shown in the left and right (resp.) panels in the bottom row 
of Figure 4. In addition, we computed extensive versions of these quantities, totaling signed and unsigned, unweighted and field-weighted divergences and curls.

Numerical simulations of coronal evolution leading to coronal mass ejections often use shearing flows (e.g., Antiochos et al. 1999) or converging flows with cancellation (e.g., Linker et al. 2003), or some combination of the two (e.g., Roussev et al. 2004), along PILs to both inject energy into the coronal magnetic field and form eruptive structures. In addition, flux emergence is expected to lead to diverging flows near PILs (see, e.g., Abbett et al. 2000). Basically, these flow patterns can be distinguished by their relationships with the local gradient in normal field: shearing velocity vectors point along normal-field contours, and converging or diverging flows near PILs are parallel or anti-parallel to normal-field gradients there. Accordingly, to characterize the presence of shearing or converging/ diverging flows near PILs in an automated way, we also decomposed flow estimates $\{\mathbf{u}\}$ into components along $\mathbf{x}_{g}$, a unit vector that points along the local horizontal gradient of $\bar{B}_{R}$,

$$
\mathbf{x}_{g} \equiv \nabla_{h} \bar{B}_{R} /\left|\nabla_{h} \bar{B}_{R}\right|
$$

and along $\mathbf{x}_{c}$, a unit vector that points along contours of $\bar{B}_{R}$,

$$
\mathbf{x}_{c} \equiv \hat{\mathbf{r}} \times \nabla_{h} \bar{B}_{R} /\left|\nabla_{h} \bar{B}_{R}\right|
$$

We denote these (signed) gradient and contour flows as $\left\{u_{g}\right\}$ and $\left\{u_{c}\right\}$, respectively. Examples of gradient and contour flows are shown in the top panel of Figure 5, as blue and red vectors, respectively. We computed $\mathcal{M}\left[u_{c}\right], \mathcal{M}\left[u_{g}\right], \mathcal{M}\left[u_{c}|d \Phi| / \Phi_{u}\right]$, and $\mathcal{M}\left[u_{g}|d \Phi| / \Phi_{u}\right]$. We also summed these quantities for each flow field to create extensive measures of flows related to shearing and convergence.

We also computed PIL-weighted contour and gradient flows, $\mathcal{M}\left[W_{P I L} u_{c} \tilde{B}_{R}\right]$, and $\mathcal{M}\left[W_{P I L} u_{c} \tilde{B}_{R}\right]$, respectively. The PIL-weighting function used here, $W_{P I L}$, was meant to identify only regions of magnetograms near PILs. It was computed in the same way as the SPIL weighting function $W_{S P I L}$ (which was used to determine $R$; see above), but with different parameters. The polarity bitmaps used for $W_{P I L}$ had a threshold of only $40 \mathrm{G}$, while the threshold for the bitmaps used to create $W_{S P I L}$ maps was $150 \mathrm{G}$; and the overlap-map of the dilated polarity-mask bitmaps was convolved with a normalized Gaussian of $\sim 7 \mathrm{Mm} \mathrm{FWHM} \mathrm{for} W_{P I L}$, versus $15 \mathrm{Mm} W_{S P I L}$. Weighting by the signed magnetic flux, $d \Phi$, introduces a cross-PIL parity, emphasizing regions with oppositely directed flows across the PIL, and deemphasizing regions where flows on opposite sides of the PIL are similar. Grayscale maps of $\left(W_{P I L} d \Phi\right)$-weighted gradient and contour flows are shown in the left and right (resp.) bottom panels of Figure 5 . In each of these bottom panels, contours of $W_{P I L}$ 
for $\bar{B}_{R}$ from the top panel are overplotted in red. For some magnetograms, $W_{P I L}=0$ everywhere, and for some others, $\left(W_{P I L} u\right)=0$ everywhere, i.e., regions with $\left|\bar{B}_{R}\right|$ above the tracking threshold and non-zero PIL-map weighting do not necessarily overlap. We note that the product of a horizontal velocity estimate with the radial magnetic field corresponds to a horizontal electric field perpendicular to both; hence, the left and right grayscale maps effectively show the components of the horizontal electric field along contours and gradients (resp.) of $\bar{B}_{R}$.

\subsection{Expected Flow Properties}

As an aside, we sought to confirm some expected correlations within the dataset. Of note, we expected diverging flows when flux was emerging (see, e.g., Abbett et al. 2000), and converging flows when flux was cancelling. Assuming that flux emergence (cancellation) leads to a positive (negative) $\dot{\Phi}$, we computed the linear and rank-order correlations between FLCT's disk-passage averaged signed, flux-weighted divergence and disk-passage averaged $\dot{\Phi} / \Phi$, and found correlation coefficients of 0.61 and 0.67 , respectively. Using DAVE's disk-passage averaged signed, flux-weighted divergence in the correlations gave values of 0.73 for both linear and rank-order correlations. We also computed the linear and rank-order correlations between the center-of-flux divergence, $D_{C O F}$, and $\dot{\Phi} / \Phi$, and found correlation coefficients of 0.45 and 0.48 , respectively.

We also looked for evidence that shearing along PILs is associated with flux emergence, as predicted by Manchester (2007). First, we correlated emerging flux with flux-weighted and unweighted measures of curl, but coefficients were in the rage 0.2-0.4 - smaller than the correlations of emergence with divergence. We also computed rank-order and linear correlations between $S_{C O F}$ and $\dot{\Phi} / \Phi$ of 0.31 and 0.29 , respectively; these are weaker than the association of $D_{C O F}$ with $\dot{\Phi} / \Phi$. In addition, the rank-order and linear correlations between disk-passage averaged, PILand flux-weighted contour flows (see the discussion below equation [19]) and diskpassage averaged $\dot{\Phi} / \Phi$ were 0.13 and 0.36 , respectively, for FLCT, and 0.24 and 0.42 for DAVE, respectively. Our measures of shearing motions were generally more weakly correlated with flux emergence than our measures of diverging motions were.

Tests with synthetic magnetograms conducted by Welsch \& Fisher (2008) with FLCT verified the supposition by Démoulin \& Berger (2003) that estimated flow magnitudes $\left|u_{c}\right|$ along contours of $\tilde{B}_{R}$ would be underestimated in comparison to flow magnitudes $\left|u_{g}\right|$ along gradients of $\bar{B}_{R}$. We did not find, however, evidence of such a 
bias in our data set: averaging over the $~ 16.5$ million pixels for which both FLCT and DAVE estimated flows, the mean and median contour speeds $\left|u_{c}\right|$ were $2 \%-5 \%$ higher than gradient speeds $\left|u_{g}\right|$ for both FLCT and DAVE. Differences between real and synthetic magnetograms might explain the discrepancy between these results: real data are more likely to possess fine-scale structure and asymmetries than synthetic data, and these structural details make it possible for tracking methods to determine the overall flow from different structures at different locations. It might still be the case, however, that our estimates are in fact biased with respect to contours / gradients in $\bar{B}_{R}$, and that true contour speeds $\left|u_{c}\right|$ exceed true gradient speeds $\left|u_{g}\right|$ by an even greater amount.

To understand the interplay between field strengths and estimated speeds, we plotted the distributions of speeds $u$ with pixel-averaged field strengths $\bar{B}_{R}$ for both FLCT and DAVE in Figure 6. The Figure includes speeds from all pixels for which both FLCT and DAVE estimates were available. Because these several million speed estimates were too clustered for a regular scatter plot, we binned speeds and field strengths, and used log-scaled shading instead of points in high-density bins, and overplotted individual points in low density bins. The log-scale shading for the FLCT plot ranges over $2.4-6.2$, and for the DAVE plot it ranges over 2.4 - 6.0. Clearly, higher speeds tend to occur in weaker fields, though a range of speeds are possible at any field strength. Statistically, however, correlations between speeds and field strengths are weak, with rank-order coefficents for FLCT and DAVE of 0.07 and -0.02 , respectively.

\section{Associating Field \& Flow Properties with Flares}

\subsection{Disk-Passage Flare Associations}

As a first step toward understanding the relationship between flows and flares (if any), we investigated the correlations between each AR's average GOES flux from flares of class $\mathrm{C}$ or greater, gross magnetogram properties (e.g., $\Phi, R$, and their time derivatives), and flow properties, averaged over the tracked component of the AR's disk passage. This coarse averaging in both space and time eliminates many detailed aspects of magnetic field structure and evolution that might be relevant to flare occurrence. Nonetheless, any correlations that remain despite this gross averaging ought to be robust. The values for flare flux used in these correlations are those listed in the right-most column of Table 1 . 
In Figures 7 and 8, we compare rank-order and linear (respectively) correlation coefficients between whole-disk-passage averaged magnetic field and flow properties, from both FLCT and DAVE, with whole-disk-passage averaged flare power from equation (1), when averaged over the 46 active regions in our study. Quantities are labeled in order of decreasing distance from $(0,0)$, the point on these plots which corresponds to complete lack of correlation with average flare power; hence, smaller numerical labels imply stronger correlation. Only zeroth, first, and second moments of magnetic field and flow properties are included in this ranking, and only the most strongly correlated properties are shown. (In plots that included higher moments, no magnetic field properties were highly-ranked enough to appear. Some higher moments of flow properties, however, did appear; but further analyses, described below, suggest that these correlations were not robust.) Odd rank numbers appear below their corresponding plot symbols, while even rank numbers appear above their symbols. To the extent that flare correlations with a given flow property from FLCT and DAVE flow agree more strongly, that flow property's plotted symbol is nearer to the dotted diagonal line in each plot (which is shown to guide the eye; it is not a fit). Quantities that do not require a flow estimate (e.g., $R$ ) lie on the diagonal line. We believe this graphical presentation of our results displays information more clearly than listing our results in tables would. Differences between linear and non-parametric, rankorder correlation coefficients could arise from lack of linearity between the correlated quantities, or non-normal variable distributions (e.g., numerous outliers), or our relatively low sample number $N_{A R}=46$, or some combination of these. It is notable, for instance, that the most highly linearly correlated quantity, $\dot{R}$, is not among the most non-parametrically correlated quantities; but its "excess kurtosis" (that relative to the normal distribution) is 15 , implying it deviates very strongly from the normal distribution.

Some of the quantities most strongly associated with our measure of disk-passage averaged flare flux, by either method of computing correlations, do not require estimating flows: $\Phi, R, \sum \tilde{B}_{R}^{2}$, and quantities involving time derivatives of the first two. Some of these magnetic field - flare correlations accord with previous work relating flare occurrence rates with total flux (Leka \& Barnes 2007; Barnes \& Leka 2008) and flux near PILs (Schrijver 2007; and also for CMEs, as reported by Falconer et al. $2003,2006)$. Table 3 shows that disk-passage averages of these quantities are correlated with each other. Schrijver (2007) has argued that large values of $R$ are more predictive of the occurrence of large flares than $\Phi$ alone, but our correlation analysis does not unequivocally support this assertion. We note that the signed, intensive rate of change of flux, $\dot{\Phi} / \Phi$, is more strongly correlated with flare power than the unsigned 
intensive rate of change, $|\dot{\Phi}| / \Phi$, implying that flux emergence is more strongly correlated with flare energy release than flux cancellation (which makes $\dot{\Phi}$ non-zero, but negative). The extensive rate of change of flux $\dot{\Phi}$ is more strongly correlated with average flare flux than the intensive rate of change of flux, $\dot{\Phi} / \Phi$, which suggests that more flare energy is released when more photospheric flux emerges than when less flux does, even if the new flux is larger relative to the pre-emergence flux.

Several extensive flow properties are significantly correlated with our measure of disk-passage averaged flare flux. Most notably, our proxy Poynting flux, $S_{R}$, is among the quantities most strongly correlated with average flare flux.

Some intensive flow properties show relatively strong negative correlation with flare flux: the averages of field-weighted unsigned vorticity, $|d \Phi(\hat{\mathbf{r}} \cdot \nabla \times \mathbf{u})| / \Phi_{u}$, unsigned divergence, $|d \Phi(\nabla \cdot \mathbf{u})| / \Phi_{u}$, and speed, $u|d \Phi| / \Phi_{u}$, among others. The magnetic flux - flare flux correlation is strong enough, however, that normalization by $\Phi_{u}$ introduces a spurious, inverse correlation between these field-weighted quantities and average flare flux. We found that the unnormalized, field-weighted versions of these average flow properties (not shown) are positively correlated with flare flux (but not sufficiently so to appear on these plots of the most strongly correlated properties).

Apart from these flux-normalized flow properties, most other intensive flow properties, averaged over the few days when ARs are near disk center, are too weakly correlated with average GOES flux to appear in these plots. We expect, however, that AR magnetic fields are more coherent on the relatively long timescale of a few days compared to flows, which have lifetimes on the order of a few hours to a day (e.g., Leighton et al. 1962). Figures 9 and 10 compare, for FLCT and DAVE, respectively, linear correlation coefficients of $\tilde{B}_{R}, u_{x}$, and $u_{y}$ in time - both frame-to-frame (thick black, blue, and red lines, resp.) and with respect to the initial frame (thin black, blue, and red lines, resp.) - for ARs 7981, 8038, and 8210. Clearly, frame-to-frame variations are much larger for estimated flows than for $\tilde{B}_{R}$, meaning flow properties tend to average incoherently - toward zero - in the disk-passage averaging we have undertaken here. This analysis suggests flows have a characteristic decorrelation timescale - taken as the time required for the autocorrelation to fall to $1 / e$ - of $\sim 6 \mathrm{hr}$.

In Figure 9, we also overplotted flare start times, shown by letters representing GOES flare class. (Note that the vertical placements of letters do not scale with actual flare fluxes.) There is no obvious correspondence between flare start times and field or flow autocorrelations. The differences between these three ARs' current-frame-to-initial-frame magnetic field correlations are intriguing, but investi- 
gating these variations is beyond the scope of this study.

\subsection{Flare Associations on Shorter Timescales}

\subsubsection{Correlation Analysis}

The flow timescale implied by the foregoing correlation analysis - $e$-folding times of $\sim 6 \mathrm{hr}$ - has implications for analyzing flow-flare relationships. Accepting the arguments of McClymont \& Fisher (1989), we presume that flows do not directly power flares, but still allow that some types of flows might tend to trigger flares. It is plausible that "trigger" flows have the same characteristic timescale, $\sim 6 \mathrm{hr}$, as the general flows in our sample. Alternatively, if some types of photospheric flows correspond to the injection of energy (perhaps via flux emergence) into the corona over several hours preceding flares (thereby being the ultimate source of flare energy, as opposed to the proximate energy source if flows were to drive flares), then there ought to be a flow-flare relationship on the coronal energy storage timescale. Work by Schrijver et al. (2005) and Longcope et al. (2005) suggests the timescale for coronal relaxation via flaring and reconnection is on the order of $24 \mathrm{hr}$. Accordingly, we now address the relationship between flow properties and the occurrence of flares in these two shorter time windows, 6 and 24 hours.

As a first step, we correlated magnetogram and flow properties with the average GOES flux from C-class and larger flares, computed via equation (1), but with the disk-passage time interval $\tau_{45}$ replaced with the flare window length, $\tau_{6}$ for 6 hours, or $\tau_{24}$ for 24 hours. For each possible window duration, two intervals were analyzed: (1) the "current" window, centered on the average time of the two magnetograms used to estimate flows; and (2) the "next" window of the same duration following the current window. For ease of reference, we label these four windows $6 \mathrm{C}, 24 \mathrm{C}, 6 \mathrm{~N}$ and $24 \mathrm{~N}$, with $\mathrm{C}$ and $\mathrm{N}$ denoting the current and next windows. To be explicit, our $6 \mathrm{C}$ window includes flares in the 6 -hour interval \pm 3 hours from each velocity estimate, and $6 \mathrm{~N}$ includes flares in the 6-hour interval 3-9 hours after that velocity estimate. The $24 \mathrm{C}$ window includes flares within \pm 12 hours of each velocity estimate, and $24 \mathrm{~N}$ includes flares over the 24-hour interval between 12-36 after that velocity estimate. In space weather parlance, forecasts corresponding to the $6 \mathrm{~N}$ and $24 \mathrm{~N}$ windows would have latencies of 3 and 12 hours, respectively. Note that our time windows do not correspond to the zero-latency forecast windows used by some other authors, e.g., Barnes et al. (2007). 
The scatter plots in Figures 11 and 12 show rank-order (R.O.) and linear correlation coefficients, for both $\mathrm{C}$ and $\mathrm{N}$ time windows, between average flare power and magnetogram or flow properties from FLCT (horizontal axis) and DAVE (vertical axis). The format of plots in these Figures is similar to that of Figures 7 and 8 . Correlated quantities are labeled in order of decreasing distance from $(0,0)$, the point on the plots which corresponds to complete lack of correlation with average flare power. Odd rank numbers appear below their corresponding plot symbols, while even rank numbers appear above their symbols. In these plots, all moments of magnetogram and flow properties were ranked, and only the ten most highly ranked properties were shown. To the extent that flare correlations with flow properties from FLCT and DAVE agree, they lie along the diagonal dotted line (not a fit). Quantities that do not require a flow estimate (e.g., $R$ ) lie on the diagonal line. No higher moments of magnetic field or flow properties beyond the zeroth, first, and second were correlated strongly enough with flare flux in these windows to appear in these plots.

These plots show relationships similar to the disk-passage averaged results. As with the disk-passage averaged correlations, many of the quantities that are strongly positively correlated with average flare power do not require estimating flows - e.g., $\Phi, R, \sum \tilde{B}_{R}^{2}$, the average unsigned field $\left\langle\left|\tilde{B}_{R}\right|\right\rangle$, as well as the standard deviations of the signed and unsigned fields, $\sigma\left[\tilde{B}_{R}\right]$ and $\sigma\left[\left|\tilde{B}_{R}\right|\right]$, respectively. Again, our proxy for the Poynting flux $S_{R}$ is among the quantities most strongly correlated with average flare flux.

The coefficient of determination is defined as $r^{2}$, where $r$ is the linear correlation coefficient. It quantifies how much of the variation in the dependent variable (avg. flare flux, here) is explained by the independent variable: when $r^{2}=1$, all of the observed variation can be explained in terms of the correlation, and a smaller value of $r^{2}$ implies less explanatory power. The largest values of $r^{2}$ for the correlations with flare flux that we performed are $\sim 0.25$, implying most variation in flare flux cannot be explained by any single variable that we considered.

The clustering of correlation coefficients from magnetic field and flow properties in Figures 11 - 12 hampers clear identification of which magnetic field and flow properties are most relevant to flare activity. Hence, relationships between these properties and flare activity should be analyzed further. 


\subsubsection{Discriminant Analysis}

We also used the discriminant analysis (DA) code developed by Barnes, Leka, and collaborators (see, e.g., Barnes et al. 2007; Leka \& Barnes 2007, 2003) to analyze our dataset. Briefly, given samples classified into distinct groups (in our case, time windows that have been classified into binary populations, either flare-associated or flare-quiet) and a set of observed quantities corresponding to each sample (in our case, magnetic field and flow properties), DA can be used to generate a discriminant function - a linear combination of the observed quantities - that, in a particular sense, best reproduces the classification of each sample into the distinct groups. This discriminant function can then be used as a way to predict the class to which a future sample will belong. While its approach of determining the linear combination of observed quantities that can explain variation in the samples is similar to that of principal component analysis, DA focuses on determining which observed quantities most strongly distinguish between the classes.

In principle, with the observed quantities standardized by subtracting their means and dividing by their variances (using, e.g., IDL's STANDARDizE.PRo), ratios of coefficients in the discriminant function quantify the relative discriminatory power of the observed quantities. Thus, DA might be used to identify the strongest discriminators, even if several of the observed quantities are correlated with each other. Values of these coefficients can, however, be unstable (e.g., Huberty \& Wisenbaker 1992), implying such ratios cannot be used to rank the importance of observed quantities with coefficients of similar magnitude. Nonetheless, DA should still be able to distinguish between variables whose coefficients differ significantly. A variable with a much smaller discriminant coefficient either inherently lacks power to accurately classify a sample, or contributes little additional discriminatory power beyond that from another variable in the discriminant function with which it is highly correlated. In accordance with these considerations, we focus less on ranking the utility of variables, versus identifying the few with strong discriminatory power in each time window.

Among other things, DA assumes the distribution of each observed quantity is normal; but Leka \& Barnes (2007) found the results of DA applied to vector magnetogram properties as predictors of solar flare occurrence to be relatively insensitive to the distributions of input properties. In addition, DA assumes that the observed quantities are independently measured. In our study, this is not strictly true for magnetogram properties like $\Phi, R$, and $\sum \tilde{B}_{R}^{2}$, which were measured from the estimated radial field $\bar{B}_{R}$ averaged from successive magnetograms; these quantities calculated 
from successive magnetogram pairs are not formally independent. The high frameto-frame correlations for the AR magnetic fields shown in Figure 9, however, imply that even if formally independent magnetogram measurements had been used, the resulting magnetic field properties would still be highly correlated.

We used DA to investigate the relationship between estimated magnetic field / flow properties and the occurrence of C-class (or stronger) flares, in each of the four time windows $(6 \mathrm{C}, 6 \mathrm{~N}, 24 \mathrm{C}, 24 \mathrm{~N})$ associated with each estimation of magnetic field and flow properties. With the nominal 96-minute cadence of estimates of magnetogram and flow properties in our study, several estimates might be associated with a single flare. Nonetheless, DA should still be able to quantify the relative power of observed magnetic field and flow properties to discriminate between flare-quiet and flare-associated windows.

While DA can identify the subset of variables with the most discriminatory power, the predictive power of that combined set of variables (with relative weights given by the discriminant function) must be evaluated separately. Climatological skill scores (see, e.g., Wheatland 2005), which quantify the improvement in forecast accuracy over the constant forecast derived from the average event frequency, can be used for this purpose. After Wheatland (2005), we denote averaging over all samples by $\langle\ldots\rangle$, the forecast probability by $f$, and the actual occurrence by $x$ (with a value of 1 or 0 for each sample, for flaring or not, respectively), with a skill score $S S$ given by

$$
S S=1-\left\langle(f-x)^{2}\right\rangle /\left\langle(\langle x\rangle-x)^{2}\right\rangle .
$$

Perfect forecasting results in $S S=1$, and scores below zero reflect worse performance than a uniform "climatological" prediction of flare likelihood equal to the observed frequency $\langle x\rangle$. While several other metrics of forecast models' predictive power exist, validation of a forecast model is not the object of this paper, so we opted not to consider additional metrics.

Which properties associated with each pair of magnetograms had the greatest discriminatory power for each time window? We note that, in addition to the magnetic field and flow properties considered in the correlation analysis above, we also considered the average flare flux during the current time windows, $6 \mathrm{C}$ and $24 \mathrm{C}$, as a property that could be included in discriminant functions for windows $6 \mathrm{~N}$ and $24 \mathrm{~N}$, respectively. Because $S_{R}$ was strongly correlated with average flare flux for many time windows in the analysis above, we started by using DA to construct separate, two-variable discriminant functions with $S_{R}$ and each of the other possible magnetic field, flow, and flare (for windows $6 \mathrm{~N}$ and $24 \mathrm{~N}$ ) variables in turn, for each of the four time windows. Separate versions of these discriminant functions were calculated 
using FLCT and DAVE flow properties. In this way, each property was separately tested "head to head" against $S_{R}$. For some windows and/ or flow estimates, it was found that $R$ (and only $R$ ) had a larger discriminant coefficient than $S_{R}$. Accordingly, for these windows, we repeated this head-to-head analysis, constructing two-variable discriminant functions with $R$ and each of the other variables in turn; and no other variable had a larger coefficient in the two-variable discriminant functions for these windows than $R$. These computations also identified the quantity that, for each window, had the most additional discriminatory power: the quantity with a discriminant coefficient closest in magnitude to the highest. For all windows but $24 \mathrm{C}, S_{R}$ and $R$ had the largest coefficients. For $24 \mathrm{C},\left\langle\left|\tilde{B}_{R}\right|\right\rangle$ - the average absolute field strength, an instrinsic magnetic quantity — had the largest coefficient relative to $S_{R}$.

We then constructed three-variable discriminant functions, separately for each additional magnetic field and flow property (also separately for FLCT and DAVE flows) beyond the two properties already identified for each time window. At this stage, the hierarchy of discriminant function coefficients differed between the two flow estimation methods for all windows, though skill scores only differed by at most 0.02 . As additional variables beyond these were considered, skill scores did not substantially increase, and the hierarchies of discriminant function coefficients constructed using variables from the two different flow estimates methods continued to disagree. Both of these facts suggested that including additional variables would result in diminishing marginal improvement in identifying magnetic field and flow properties associated with flares. Accordingly, we did not pursue this analysis further.

Table 4 summarizes the outcome of our discriminant analysis. Time windows are listed in the left-most column, and method used to estimate flows are listed in the next column. The variables used to construct each discriminant function are shown in the next column. For multiple-variable discriminant functions, the discriminant coefficients of each standardized variable are listed in square brackets. These results suggest that $S_{R}$ and $R$ consistently have strong discriminatory power between flaring and non-flaring populations, regardless of tracking method used to estimate $S_{R}$. Further, given their commensurate discriminant coefficients, our discriminant analysis implies that these two parameters each possess some unique predictive capability. The (intensive) average unsigned field strength, $\left\langle\left|\tilde{B}_{R}\right|\right\rangle$, also had relatively large discriminatory power in many cases.

The next four columns of Table 4 show predicted and actual flare frequencies for each window considered. (There are many more flares in the statistics listed in these columns - from around a hundred to a few hundred, depending on the window 
— than are listed in $\S 2$. This discrepancy results from "overlapping measurements": many average magnetic fields and flow estimates having a common flare in their windows.) In classifying events for each window, we used the same data that were used to construct the discrimant function for that window, which tends to bias the success rate upward (Hills 1966). (Unbiased calculations require constructing separate discriminant functions with each sample removed, and using the resulting discriminant function to classify the removed sample. This is both time consuming, and, in tests we performed, did not significantly alter event classifications.) The final column lists the climatological skill scores, $S S$, derived for the corresponding discriminant function. For comparison with the skill scores reported here, Barnes et al. (2007) used a large set of properties of AR magnetic fields derived from vector magnetograms, and achieved an $S S$ value of 0.35 for predicting C-class or larger flares in the 24 hours following each magnetogram. Our differing definition of the future 24 hour window (12-hour latency in our case, no latency in theirs) precludes direct comparison of our results with theirs, but their results nonetheless provide useful context.

We also note that the vector magnetograms used by Barnes et al. (2007) are probably superior to LOS magnetograms for computing radial magnetic field strengths. For instance, Knoll et al. (2008) computed the skill scores for single-variable discriminant functions using total unsigned flux, computed from cosine-corrected LOS magnetograms at various distances from disk center; they found that the skill score using magnetograms within $45^{\circ}$ of disk center (as with our data set) was a factor of three lower than the skill score using only magnetograms from within $10^{\circ}$ of disk center. This demonstrates that using off-center LOS data to estimate $B_{r}$, as we have done, can negatively impact skill scores.

Skill scores were negative for the $6 \mathrm{~N}$ window using our discriminant functions, implying no skill beyond that based upon event frequencies in the data set. As flares are infrequent, forecasting for small time windows tends to be inaccurate, as a short time interval is more likely than not to be flare quiet (Leka \& Barnes 2007); this might partly explain why forecast skill for window $24 \mathrm{~N}$ exceeded that of $6 \mathrm{~N}$. For window $24 \mathrm{~N}$, flare flux averaged over the current 24 hour window, $\mathcal{F}_{24 \text {,current }}$, exhibited some discriminatory power, and evidently shares some predictive power with $R$, as the latter's coefficient shrank when the former was included in the discriminant function. The tendency for regions that have already flared to flare again soon after is referred to as persistence in the flare forecast literature (Zirin \& Marquette 1991).

In Table 5, we list statistical properties of the two variables for each time window with the highest discriminant function coefficients from Table 4. We first list 
the means of the raw variables for the flaring and non-flaring populations from each window, separately and combined; and then list the corresponding standard deviations of each variable's combined flaring and non-flaring populations. We then list the means of the standardized versions of each variable for the flaring and non-flaring populations. Results from FLCT were used for the only quantity that involves flows, $S_{R}$.

\section{Discussion \& Conclusions}

Magnetic energy is the predominant form of energy in the low corona (Forbes 2000), and is thought to power flares and CMEs. This energy is thought to emerge into the corona in active region magnetic fields (McClymont \& Fisher 1989). As the photospheric magnetic fields should be strongly coupled to the plasma (e.g., Parker 1984), the transport of magnetic energy across the photosphere should be governed by equation (16): either radial (vertical) flows transport current-carrying magnetic flux across the photosphere (e.g., Leka et al. 1996; Abbett \& Fisher 2003), or photospheric flows act on already emerged fields (e.g., Brown et al. 2003; Antiochos et al. 1999). Accurate estimates of photospheric flows, therefore, could be used to study physical processes that lead to the buildup of coronal magnetic energy that is released in flares and CMEs. If processes that trigger flares/ CMEs are related to photospheric evolution, then studying photospheric flows can improve our understanding of these processes. It is possible, however, that trigger processes are essentially coronal, and only related to photospheric evolution in a statistical sense. In the latter case, knowledge about trigger processes that could be gleaned from studies of photospheric evolution would be limited.

In this study, we attempted to relate properties of magnetic fields and flow fields estimated by tracking photospheric magnetograms to the release of coronal magnetic energy in the form of flares. We distinguished between extensive properties, which scale with AR size, and intensive properties, which do not depend directly upon AR size. Using correlation analysis, we identified several properties that were roughly equally associated with flare flux averaged over several time windows. To distinguish which among these properties was most strongly related to the occurrence of flares, we employed linear discriminant analysis. Using both methods, we found one extensive

flow property, $S_{R}=\sum u \tilde{B}_{R}^{2}$, to be associated with flare flux and flare occurrence at a level comparable to the most strongly flare-associated magnetic field property, $R$, which quantifies the flux near strong-field polarity inversion lines (SPILs). The 
association of $S_{R}$ with flare activity was robust regardless of whether FLCT or DAVE were used to estimate flows, and for a variety of time windows corresponding to each flow estimate. To quantify the similarity between the flows when weighted by $\tilde{B}_{R}^{2}$, we computed the linear and rank-order correlation coefficients between $u_{\mathrm{FLCT}} \tilde{B}_{R}^{2}$ and $u_{\text {DAVE }} \tilde{B}_{R}^{2}$ for the $\sim 16.5$ million pixels where both estimated flows; they were 0.89 and 0.91 , respectively. We note that $S_{R}$ was more strongly associated with flares than $\sum \tilde{B}_{R}^{2}$, implying the square of the magnetic field strength is not solely responsible for association. We also found $u$ and $\left|\bar{B}_{R}\right|$ to be uncorrelated with each other, so the association with flaring does not arise from a hidden correlation between $u$ and $\left|\bar{B}_{R}\right|$. Hence, we conclude that flow information is an essential factor in the association between $S_{R}$ and flare activity that we have identified. We could not unambiguously associate any intensive property of flows with flare activity, a potentially significant fact we discuss in more detail below.

The extensive or intensive scaling of flare-associated magnetic field and flow properties is relevant to flare and CME processes. Triggers of flares and CMEs might be small-scale features like current sheets; for instance, globally significant reconnection begins at current sheets in the breakout model of Antiochos et al. (1999) and the tether cutting model of Moore et al. (2001). In contrast, the non-potential magnetic structures associated with flares and CMEs, such as filaments and sigmoids, are largescale features. Free magnetic energy is probably stored on large scales, because the difference between the actual and potential magnetic fields is non-local. For example, the presence of a current sheet - a compact non-potential structure - can alter the magnetic field far from the current sheet itself. Flares occur over a range of energies (e.g., Hudson 1991), and larger ARs can produce larger flares (Sammis et al. 2000; Kucera et al. 1997), suggesting that peak flare energy is an extensive property of ARs. The effectively exponential weighting of average flare flux by flare class in equation (1) means that the average flare fluxes we used here strongly emphasize the largest flares. Hence, our measure of flare activity essentially scales extensively. Consequently, it is perhaps unsurprising that the AR properties we found to be most strongly associated with flare activity are extensive.

Figure 13 illustrates this extensive scaling. The squares plot the relationship between the log of the average flux from flares at or above GOES C1.0 level over the time interval $\tau_{45}$ when each AR was within $45^{\circ}$ of disk center, versus the log of the average total unsigned magnetic flux in these ARs over that time. Error bars show RMS variance in flux for each AR over $\tau_{45}$; errors expected from $20 \mathrm{G}$ per pixel noise in an individual MDI magnetogram are about the size of the plotted squares or smaller. Triangles show the flux in the 22 ARs that did not flare at or above GOES C1.0 level. 
(Because many A- and B-class flares are not reliably ascribed to a source AR in the GOES catalog, emission from these flares has been excluded.) It is clear the squares show a trend, which we crudely quantified by computing linear and rank-order (R.O.) correlations between magnetic flux and average flux from flares above the GOES C1.0 level, both for raw values using all ARs, and for logarithms of these values using only ARs that flared above this level. We also computed a least- absolute- deviation fit of the $\log$ of average flare flux as a function of the log of unsigned magnetic flux, which yielded a slope of 1.40. While our small sample size hampers our ability rigorously characterize this relationship, we note that the value of the slope differs from the value of $\sim 1.15$ found by Pevtsov et al. (2003) to relate total unsigned flux to thermal soft X-ray (SXR) luminosity. A discrepancy between the scalings of thermal and non-thermal (flare) SXR luminosities with AR magnetic flux might indicate that different physical processes generate each type of emission. Also, the magnetic flux - flare flux correlation might account for some of the variability in AR flare productivity reported in other studies relating flares to magnetic field properties and/ or evolution - e.g., Abramenko (2005) and LaBonte et al. 2007. An intensive measure of AR flare productivity might be formed by normalizing average AR flare flux by AR magnetic flux $\Phi$ raised to some power $a$; for a larger sample, one could follow the procedure adopted by Fisher et al. (1998) to determine an exponent that removed any correlation between average flare flux and magnetic flux. Linearity in the magnetic flux - flare flux relationship could be consistent with uniform free energy input per unit flux (uniform "specific free magnetic energy"). Nonlinearity might reflect magnetic interactions after emergence (as would occur in an avalanche model of flares, Lu \& Hamilton 1991), or an aspect of the processes (dynamo, emgergence) that produce active region magnetic fields.

Given $R$ 's extensive scaling, it is interesting that Schrijver (2007) found that ARs with similar values of $R$ could produce flares with a range of energies. This suggests that the amount of flux near SPILs does not directly imply the amount of energy that will be released in any subsequent flares. It is possible that $R$ scales with the total amount of magnetic energy available to be released, but that the size of the actual energy released depends upon the details of the coronal magnetic field configuration. Welsch \& Li (2008) found that $R$ tended to increase while total AR flux was increasing, implying that concentrations of flux near SPILs tend to arise by the emergence of flux, as opposed to the convergence of already emerged flux. Emergence has long been supposed to be a trigger of flares (e.g., Priest \& Heyvaerts 1974). So, despite its extensive scaling, a large value of $R$ might indicate the presence of a flare trigger, as well as the total amount of free magnetic energy present in a 
configuration.

The flow property most strongly associated with average flare flux, $S_{R}$, has units of an energy flux: energy per unit area per unit time. In Table 5, raw values for this quantity are $\sim 10^{7} \mathrm{~km} \mathrm{G}^{2} \mathrm{sec}^{-1}$; mulitplying by both $10^{5}$, to convert $\mathrm{km}$ to $\mathrm{cm}$, and the MDI disk-center pixel area, $d a \simeq 2 \times 10^{16} \mathrm{~cm}^{2}$, and dividing by $4 \pi$ (for consistency with the Poynting flux in equation [17]) gives a power on the order of $10^{27} \mathrm{erg} \mathrm{sec}^{-1}$. Integrated over the $\sim 10^{5}$ seconds in a day or so, this represents the transport of $\sim 10^{32}$ ergs day $^{-1}$ of magnetic energy. While we have referred to $S_{R}$ as a proxy for the integrated Poynting flux of magnetic energy across the photosphere, actual correlation between these quantities has not been demonstrated. If the true Poynting flux were an order of magnitude smaller, so $\sim 10^{31} \mathrm{ergs} \mathrm{day}^{-1}$, this would represent a enough magnetic energy to power a large flare. This would contradict the view advocated by McClymont \& Fisher (1989), who argued that even organized, nearsurface flows could not inject enough free magnetic energy into the coronal field to power large flares. Instead, they suggested that the magnetic energy that is released in very large flares must be carried into the corona with magnetic fields as they emerge.

The view that flares are powered by free magnetic energy that emerges with AR fields is, however, supported by the fact that active regions which have recently flared are more likely flare again, compared to active regions that have not recently flared. This phenomenon has been referred to as persistence (Zirin \& Marquette 1991). The alternative view requires post-emergence energy buildup in the corona, and is plausible: flows could act on already emerged fields, leading to the storage of free magnetic energy in the corona, which could then be released in a flare. But why would flows preferentially inject magnetic energy into some ARs, making these flare more frequently, and not into other ARs? Indeed, it seems that some ARs emerge carrying a lot free magnetic energy, which is released in many flares; such ARs might be "born bad" (e.g., Zirin \& Liggett 1987).

But if flare-prone ARs are born bad, why is average flare flux correlated with any photospheric flow property? It could be that $S_{R}$ is in some way related to the emergence of new, non-potential magnetic fields. But if emergence were truly the dominant manner of free energy injection into the corona, then $\dot{\Phi}$ would be the more fundamental magnetic field property, and therefore presumably a better predictor of average flare flux. (Perhaps our timescale for computing $\dot{\Phi}$ - nominally, 96 minutes - is too short; see below for more on this point.) It could be that those ARs that emerge with the largest amount of free magnetic energy, due to the presence of electric currents, are also the most likely to exhibit large flows — perhaps as a result 
of Lorentz forces arising from these currents.

In addition, it is possible that cancellation, driven by seemingly random flows, might lead to the buildup of free magnetic energy in the corona, which might then be released in flares and CMEs. This process was not considered by McClymont \& Fisher (1989). Turbulent convective flows tend to systematically drive separate photospheric magnetic features into close proximity in downflow lanes between supergranules (e.g., Schrijver et al. 1997); while same-sign features simply merge, opposite-sign features undergo cancellation. Barring unrealistic symmetry, such cancellation generally forces reconnection between coronal fields, which has the effect of "stranding" any magnetic helicity that was present in those fields in the corona, and tends to increase the magnetic free energy of the coronal field (Welsch 2006). The buildup of free magnetic energy by this process, however, might be too slow to explain many large flares, which can recur frequently in the same active region.

We were unable to clearly associate any intensive properties of flows with the occurrence of flares. Taken at face value, this might suggest that the flare mechanism itself is fundamentally extensive, as in the avalanche model of Lu \& Hamilton (1991), for example. We must, however, qualify this result in several ways. As discussed above, our measure of flaring activity is extensive, which probably biases our analysis against detecting relationships between intensive flow properties and flares. In addition, our flow estimates are almost certainly subject to serious systematic errors. Tests of FLCT, DAVE, and other flow estimation techniques by Welsch et al. (2007) with sequences of synthetic magnetograms from MHD simulations, in which the true velocities are known, showed that both FLCT and DAVE systematically underestimated the fluxes of magnetic helicity and energy. (These shortcomings led to the development of DAVE4VM [Schuck 2008], which uses information about the horizontal magnetic field - unavailable in this study — to make improved estimates of flows and the fluxes of magnetic energy and helicity.) Moreover, these tests also showed that FLCT systematically underestimated flow speeds. So the tracking methods and/ or data (2" spatial resolution, 96-min. cadence) employed in this study might be too limited to reveal any intensive flow patterns that are associated with flares. Or it might be that intensive flow properties are important, in some cases, for increasing coronal free magnetic energy, and/ or for triggering flares, but that the efficacy of a given flow pattern at injecting significant energy or triggering flares depends upon the details of the particular photospheric and coronal magnetic field configuration. In this case, statistical relationships between flows and flares would be hard to identify. This last possibility motivates development of time-dependent models of coronal magnetic field evolution, driven by magnetograms, as tools necessary to understand 
(and eventually predict) the coronal response to photospheric flows.

Could it be that some flow patterns are, in fact, anti-correlated with flare onset? Some previous results have suggested an inverse correlation between flow vorticity and flare occurrence. In his talk at the SHINE meeting in 2007, A. A. Pevtsov presented preliminary results suggesting that flares are more likely to occur after a time interval in which sunspots were observed to rotate. He presented a basic physical picture to explain these observations: (1) the solar interior acts as a current driver that imposes a fixed amount of twist (equivalently, current) on the flux systems that comprise active regions; (2) if the twist in the coronal portion of a flux system does not match the twist on the portion in the interior, twist will propagate to equilibrate the two portions (Longcope \& Welsch 2000); (3) the twist propagation will be seen as a rotation of photospheric footpoints, which will slow as the twist gradient across the photosphere shrinks; (4) the twist injected into the corona implies that free magnetic energy is present in the corona, which will be released in a flare or flares. Based upon this model, one should expect that, statistically, flares will be more likely when the corona has more twist, which should occur when footpoint rotation has begun to slow or has ceased.

This preliminary investigation of the statistical relationships between flows and flares has provided useful insights. We have investigated the properties of AR photospheric flows, characterized the correlation times of AR fields and flows, and identified a quantity, $S_{R}$, that might be useful for flare prediction.

Further study will be necessary to fully understand the role of photospheric evolution in solar flares. A study similar to that conducted here, but with a larger sample of ARs, could verify that our results are not statistical aberrations, due to our small sample size. Also, our tracking techniques are primarily senstive to flows on the spatial scale of the windowing parameter used by DAVE and FLCT, $\sim 17^{\prime \prime}$. Flow-flare relationships on smaller and larger spatial scales should also be investigated; perhaps particular flows on very small scales, or coherent flows on large scales, are important.

In addition, evolution on other time scales should also be investigated. Our correlation analysis suggested that flux emergence, quantified by the change in flux $\dot{\Phi}$ over our nominal 96-minute magnetogram cadence, was correlated with average flare flux, but not as strongly as other field and flow properties that we considered. But fractional changes of flux in magnetograms are not large on this time scale, which is short relative to the characteristic hours-long emergence timescale of active region flux. Consequently, the statistical relationship between flux emergence and flares on longer time scales (e.g., 6 - 48 hours) could reveal stronger correlations with 
flare activity. In addition, the decorrelation rate of magnetograms (see Figs. 9 10) separated in time by intervals longer than our nominal cadence could provide a quantitative measure of AR magnetic evolution that might also be correlated with flares. Work on these efforts is underway.

The authors appreciate the referee's careful reading of the manuscript and thoughtful comments, which have improved the manuscript. B. T. Welsch and Y. Li acknowledge the support of NSF grant ATM-0451438, and NSF SHINE award ATM-0752597 for B. T. W. Y. L. additionally acknowledges support from NSF CISM ATM-0120950. P. W. Schuck acknowledges support from NASA LWS TR\&T grant NNH06AD87I, LWS TR\&T Strategic Capability grant NNH07AG26I, and ONR. G. H. Fisher acknowledges support for his development work on FLCT from NSF awards ATM0641303 and ATM-0551084. The discriminant analysis code we used was kindly supplied by G. Barnes and K. D. Leka, who received funding from AFOSR under contracts F49620-00-C-0004, F49620-03-C-0019, and from NASA under contract NNH07CD25C. We thank the SOHO/MDI team for making their database available and easy to use. MDI is funded through NASA's Solar and Heliospheric Physics program. The SOHO project results from international cooperation between NASA and ESA. B. T. W. also gratefully acknowledges discussions with Dr. Maureen Lahiff regarding statistical analysis techniques.

\section{A. Mercator Projections}

A map projection is a two-dimensional, planar representation of a two-dimensional, spherical surface. In solar physics, instruments typically record a two-dimensional image of the Sun in some wavelength range as a plane-of-sky (POS) map of intensity variations. When the recorded emission originates in a relatively narrow atmospheric layer - as in a typical photospheric magnetogram, for instance - this procedure amounts to a projection from the imaged, spherical atmospheric layer on the observer's side of the Sun onto the image plane. From the viewpoint of scientific analysis, this projection poses many problems; for instance, foreshortening distorts spatial relationships between image features.

To ameliorate this and other undesirable properties of the POS projection, the POS positions of image data points can be converted to latitude and longitude in some heliographic coordinate system. The origin of the Stonyhurst heliographic coordinate system (HCS), for instance, is at the intersection of the apparent solar equator and the 
central meridian as seen from Earth (Thompson 2006). This conversion from POS to HCS coordinates is a deprojection. Unfortunately, after transformation into longitude and latitude coordinates $(\phi, \tilde{\theta})$ for a given heliographic coordinate system, data that were regularly spaced in the POS projection are typically not regularly sampled in $(\phi, \tilde{\theta})$. (We use $\tilde{\theta}$ for latitude, to distinguish it from the co-latitude $\theta$ typically used in spherical polar coordinates.) Consequently, analyses of spatial relationships between image features are still problematic.

Quantitative analyses of the spatial properties of irregularly sampled data lying on a spherical surface therefore require interpolation of these data onto a regular coordinate system. While interpolation onto spherical gridpoints uniformly spaced in $(\phi, \tilde{\theta})$ is possible, both the distances and directions between nearby gridpoints in such systems vary in complex ways in different regions of the grid. Instead, we have opted to use the Mercator projection to convert the data from $(\phi, \tilde{\theta})$ to a rectilinear coordinate system. Often this two-projection procedure - deprojecting from the POS coordinates in the image plane to the HCS, and then transforming the data again by interpolating onto spherical points corresponding to a uniform grid - is loosely referred to as "deprojecting" the data, even though it is probably best described as a reprojection. In the discussion that follows, we assume the HCS positions of the POS image points have already been computed, using, e.g., the IDL functions XY2LONLAT.PRO in SSWIDL's /ssw/gen/idl/solar/ .

We chose the Mercator projection because it is conformal: locally, it does not distort shape. This means the Mercator projection should be well-suited for unbiased (in direction) tracking of features in image sequences, or any study of spatial variations in image structure, e.g., the magnitude and direction of intensity gradients. But, since any projection of a spherical surface onto a plane must distort either shape or scale (if not both!), the pixel scale varies over the projected image. (Equivalently, this projection is non-authalic, or not area-preserving.) We have developed our Mercator projection procedure for tracking studies, on the assumption that correcting distortion of scale is easier than correcting directional bias in tracked displacements introduced by a non-conformal deprojection.

The distortion of scale in global Mercator projections is a function of latitude alone. The horizontal Mercator coordinate, $x$, is mapped one-to-one with the heliocentric longitude, $\phi$, and is independent of latitude. Because the physical distance between lines of constant longitude decreases as $\cos (\tilde{\theta})$ with increasing latitude, the projection's scale (the actual distance corresponding to a fixed distance in the reprojected image) must decrease with increasing latitude. Displacements in the vertical 
Mercator coordinate, $d y$, corresponding to a fixed latitudinal displacement, $d \tilde{\theta}$, increase toward the poles. Quantitatively, $y$ is related to latitude $\tilde{\theta}$, via

$$
y=\ln (\tan (\tilde{\theta})+\sec (\tilde{\theta}))
$$

As $\tilde{\theta} \rightarrow \pi / 2$, therefore, $y$ diverges logarithmically. Differentially, $d y=\sec (\tilde{\theta}) d \tilde{\theta}$, and the $d y$ corresponding to an interval $d \tilde{\theta}$ also diverges toward the pole. To compensate for these distortions, small distances - e.g., tracked displacements, or the separations between pixel centers when computing gradients - must be multiplied by a factor of cosine latitude, $\cos (\tilde{\theta})$, when converting Mercator distances to physical distances.

It is desirable to preserve the spatial resolution in the original data set. Typically, this means the pixel width, $\Delta s$, in the projected data should match the pixel size at the sub-observation point (disk center) in the POS data. Hence, this fixes the spatial sampling rate of the original image that must be used to interpolate the POS data onto the Mercator grid. The number of necessary longitudinal samples for an interval of longitude $\Delta \phi$ is the horizontal distance corresponding to this interval, $L_{\phi}=R_{\odot} \Delta \phi$, divided by the original pixel size, $\Delta s$.

To preserve spatial resolution in the latitidunal direction is more complex. For a given interval of latitude, $\Delta \tilde{\theta}=\tilde{\theta}_{f}-\tilde{\theta}_{i}$, the interval $\Delta y=y_{f}-y_{i}$ is given by

$$
\Delta y=\ln \left(\frac{\tan \left(\tilde{\theta}_{f}\right)+\sec \left(\tilde{\theta}_{f}\right)}{\tan \left(\tilde{\theta}_{i}\right)+\sec \left(\tilde{\theta}_{i}\right)}\right) \text {. }
$$

The number of spatial samples necessary to preserve the initial pixel scale $\Delta s$ is therefore the distance corresponding to the interval of latitude, $L_{\tilde{\theta}}=R_{\odot} \Delta \tilde{\theta}$, divided by $\Delta s$, and multiplied by the ratio $\Delta y / \Delta \tilde{\theta}$. For $\Delta \tilde{\theta} \in\left\{30^{\circ}, 45^{\circ}, 75^{\circ}\right\}$, the ratios $\Delta y / \Delta \tilde{\theta}$ are $\{1.05,1.12,2.02\}$, respectively. Use of too few samples in the vertical direction would distort shapes in the image, and destroy the projection's conformal properties.

The necessary interpolation is done by first computing the Delaunay triangulation of the irregularly sampled data in (latitude-longitude), then interpolating onto the latitude-longitude points corresponding to the regular Mercator grid of the output array. (This output grid is rectilinear in [latitude-longitude], but not regularly spaced in latitude.)

When deprojecting sequences of image data, we found that small changes in instrument pointing could lead to changes of $+/-1$ pixel when computing array sizes from pointing information in the header files. Consequently, we fixed the output 
arrays for an entire image sequence, as opposed to determining these separately for each image.

To verify that the algorithm worked properly, we projected latitudinal and longitudinal bands onto a POS image of a sphere, and then reprojected the POS image backward.

With the availability of full-disk vector magnetogram data from SOLIS/VSM, SDO/HMI, and other instruments, conformal projections could find wider application. To view data near the poles without distortion seen in typical global-scale Mercator projections (in which the line of tangency between the sphere and projection cylinder lies along the equator), local Mercator projections (in which the line of tangency runs through the center of the deprojected region) could be used, in a manner similar to the universal transverse Mercator (UTM) approach used in terrestrial maps, e.g., NATO topographical maps. 
Table 1. Active Region Sample

\begin{tabular}{|c|c|c|c|c|}
\hline $\begin{array}{l}\text { NOAA } \\
\text { AR \# }\end{array}$ & Start Time & Stop Time & $\begin{array}{l}\text { Avg. GOES Flux } \\
\left(\mu \mathrm{W} \mathrm{m}^{-2} \text { day }^{-1}\right)\end{array}$ & $\begin{array}{l}\text { Avg. GOES Flux }{ }^{\mathrm{a}},>\mathrm{C}^{\mathrm{b}} \\
\quad\left(\mu \mathrm{W} \mathrm{m}^{-2} \text { day }^{-1}\right)\end{array}$ \\
\hline 7981 & 1996-08-01, 04:19 & 1996-08-04, 22:24 & 0.8 & 0.0 \\
\hline 7986 & $1996-08-28,04: 51$ & 1996-08-31, 22:24 & 0.4 & 0.0 \\
\hline 7997 & $1996-11-22,13: 03$ & $1996-11-23,17: 39$ & 0.0 & 0.0 \\
\hline 7999 & $1996-11-24,06: 27$ & $1996-11-28,20: 48$ & 8.7 & 8.0 \\
\hline 8003 & 1996-12-08, 01:36 & $1996-12-11,12: 48$ & 0.5 & 0.0 \\
\hline 8004 & $1996-12-15,08: 03$ & $1996-12-20,06: 27$ & 0.1 & 0.0 \\
\hline 8005 & $1996-12-16,14: 24$ & $1996-12-21,12: 51$ & 0.6 & 0.5 \\
\hline 8006 & $1996-12-17,08: 00$ & $1996-12-21,15: 59$ & 0.0 & 0.0 \\
\hline 8015 & 1997-01-30, 03:15 & $1997-02-03,22: 24$ & 0.3 & 0.0 \\
\hline 8020 & $1997-03-10,20: 48$ & $1997-03-15,03: 12$ & 0.0 & 0.0 \\
\hline 8038 & $1997-05-09,12: 52$ & $1997-05-14,00: 04$ & 0.3 & 0.3 \\
\hline 8040 & 1997-05-18, 08:04 & $1997-05-23,12: 52$ & 3.1 & 2.9 \\
\hline 8052 & $1997-06-13,14: 24$ & $1997-06-17,22: 24$ & 0.0 & 0.0 \\
\hline 8076 & 1997-08-28, 03:11 & 1997-09-01, 09:39 & 2.5 & 1.9 \\
\hline 8078 & 1997-08-29, 01:39 & 1997-09-01, 22:24 & 0.0 & 0.0 \\
\hline 8081 & 1997-09-05, 01:39 & 1997-09-09, 12:48 & 0.0 & 0.0 \\
\hline 8082 & 1997-09-05, 01:39 & 1997-09-09, 23:59 & 0.2 & 0.0 \\
\hline 8083 & $1997-09-06,14: 23$ & $1997-09-09,12: 48$ & 3.7 & 3.3 \\
\hline 8084 & 1997-09-08, 09:39 & $1997-09-13,12: 48$ & 1.0 & 0.6 \\
\hline 8085 & 1997-09-12, 01:36 & $1997-09-14,06: 24$ & 1.4 & 1.2 \\
\hline 8086 & $1997-09-15,14: 27$ & $1997-09-20,12: 51$ & 1.4 & 1.2 \\
\hline 8088 & 1997-09-24, 08:03 & $1997-09-26,22: 24$ & 45.4 & 44.2 \\
\hline 8087 & 1997-09-26, 17:36 & 1997-09-29, 03:12 & 0.0 & 0.0 \\
\hline 8090 & $1997-10-05,11: 15$ & $1997-10-07,06: 27$ & 0.0 & 0.0 \\
\hline 8093 & $1997-10-10,17: 35$ & $1997-10-15,12: 51$ & 0.0 & 0.0 \\
\hline 8096 & $1997-10-16,17: 35$ & 1997-10-22, 08:03 & 0.0 & 0.0 \\
\hline 8097 & 1997-10-19, 19:11 & $1997-10-24,16: 00$ & 0.7 & 0.7 \\
\hline 8100 & 1997-10-31, 01:39 & $1997-11-03,23: 59$ & 88.6 & 87.2 \\
\hline 8108 & $1997-11-17,22: 23$ & $1997-11-22,06: 23$ & 3.1 & 1.6 \\
\hline 8123 & $1997-12-17,01: 39$ & $1997-12-21,22: 17$ & 0.1 & 0.0 \\
\hline 8135 & 1998-01-11, 17:36 & $1998-01-15,20: 48$ & 0.0 & 0.0 \\
\hline 8156 & 1998-02-13, $17: 36$ & $1998-02-18,22: 24$ & 3.9 & 2.8 \\
\hline
\end{tabular}


Table 1-Continued

\begin{tabular}{ccccc}
\hline \hline $\begin{array}{c}\text { NOAA } \\
\text { AR \# }\end{array}$ & Start Time & Stop Time & $\begin{array}{c}\text { Avg. GOES Flux } \\
\left(\mu \mathrm{W} \mathrm{m}^{-2} \text { day }^{-1}\right)\end{array}$ & $\begin{array}{c}\text { Avg. GOES Flux } \\
\left(\mu \mathrm{W} \mathrm{m}^{-2} \text { day }^{-1}\right)\end{array}$ \\
\hline 8158 & $1998-02-15,01: 36$ & $1998-02-18,22: 24$ & 0.1 & 0.0 \\
8179 & $1998-03-13,03: 12$ & $1998-03-17,22: 24$ & 20.6 & 19.7 \\
8210 & $1998-04-28,17: 39$ & $1998-05-03,16: 00$ & 46.4 & 45.2 \\
8214 & $1998-05-02,17: 36$ & $1998-05-05,16: 00$ & 11.2 & 10.7 \\
8218 & $1998-05-10,03: 15$ & $1998-05-14,22: 24$ & 2.5 & 1.5 \\
8222 & $1998-05-15,17: 36$ & $1998-05-19,03: 15$ & 0.5 & 0.5 \\
8220 & $1998-05-15,14: 24$ & $1998-05-19,09: 39$ & 0.6 & 0.6 \\
8227 & $1998-05-31,01: 39$ & $1998-06-02,12: 51$ & 0.0 & 0.0 \\
8230 & $1998-06-02,11: 15$ & $1998-06-06,22: 24$ & 0.0 & 0.0 \\
8243 & $1998-06-17,01: 36$ & $1998-06-21,12: 48$ & 4.6 & 19.4 \\
8375 & $1998-11-02,03: 11$ & $1998-11-06,22: 24$ & 20.0 & 0.0 \\
8377 & $1998-11-07,03: 12$ & $1998-11-09,19: 15$ & 0.0 & 0.6 \\
8392 & $1998-11-26,01: 36$ & $1998-11-29,12: 48$ & 2.6 & 0.4 \\
8393 & $1998-11-28,01: 36$ & $1998-12-01,22: 24$ & 0.4 & \\
\hline
\end{tabular}

aAfter Abramenko (2005)

b " $>$ C" denotes only flare with peak flux $>$ GOES C1.0 level 
Table 2. Magnetic Field and Flow Properties Calculated

\begin{tabular}{|c|c|c|c|}
\hline Property & Scaling ${ }^{\mathrm{a}}$ & Related Physical Quantity & Citation $(\mathrm{s})^{\mathrm{b}}$ \\
\hline$\Phi$ & $\mathrm{E}$ & Quantify AR "magnetic size" & $\begin{array}{l}\text { Fisher et al. 1998; } \\
\text { Leka \& Barnes 2003 }\end{array}$ \\
\hline$\dot{\Phi}$ & $\mathrm{E}$ & Flux emergence, cancellation & $\begin{array}{l}\text { Priest \& Heyvaerts 1974; } \\
\text { Linker et al. } 2003\end{array}$ \\
\hline$\dot{\Phi} / \Phi$ & I & Flux emergence, cancellation & $"$ \\
\hline$|\dot{\Phi}| / \Phi$ & I & Unsigned flux changes & $"$ \\
\hline $\mathcal{M}\left[\tilde{B}_{R}\right]$ & I & Field moments & Leka \& Barnes 2003 \\
\hline $\mathcal{M}\left[\left|\tilde{B}_{R}\right|\right]$ & $\mathrm{I}$ & Unsigned field moments & $"$ \\
\hline $\mathcal{M}[u]$ & I & Flow moments & - \\
\hline $\mathcal{M}\left[u|d \Phi| / \Phi_{u}\right]$ & I & Flux-weighted flow moments & - \\
\hline$\sum u$ & $\mathrm{E}$ & Amount of motion in AR & - \\
\hline$\sum u|d \Phi|$ & $\mathrm{E}$ & Unsigned flux-weighted speeds & - \\
\hline $\mathcal{M}[|\hat{\mathbf{r}} \cdot \nabla \times \mathbf{u}|]$ & I & Unsigned vorticity & Brown et al. 2003 \\
\hline $\mathcal{M}\left[|d \Phi(\hat{\mathbf{r}} \cdot \nabla \times \mathbf{u})| / \Phi_{u}\right]$ & $\mathrm{I}$ & Flux-weighted unsigned vorticity & $"$ \\
\hline$\sum \hat{\mathbf{r}} \cdot(\nabla \times \mathbf{u})$ & $\mathrm{E}$ & Net vorticity & $"$ \\
\hline$\sum|\hat{\mathbf{r}} \cdot(\nabla \times \mathbf{u})|$ & $\mathrm{E}$ & Unsigned vorticity & $"$ \\
\hline$\sum|d \Phi|(\hat{\mathbf{r}} \cdot \nabla \times \mathbf{u})$ & $\mathrm{E}$ & Flux-weighted vorticity & $"$ \\
\hline$\sum|d \Phi(\hat{\mathbf{r}} \cdot \nabla \times \mathbf{u})|$ & $\mathrm{E}$ & Unsigned flux-weighted vorticity & $"$ \\
\hline $\mathcal{M}[\nabla \cdot \mathbf{u}]$ & $\mathrm{I}$ & Converging/diverging flows & $\begin{array}{l}\text { Linker et al. 2003; } \\
\text { Wang } 2006\end{array}$ \\
\hline $\mathcal{M}[|\nabla \cdot \mathbf{u}|]$ & I & Unsigned conv./div. flows & $"$ \\
\hline $\mathcal{M}\left[|d \Phi|(\nabla \cdot \mathbf{u}) / \Phi_{u}\right]$ & I & Flux-weighted conv./div. flows & $"$ \\
\hline $\mathcal{M}\left[|d \Phi(\nabla \cdot \mathbf{u})| / \Phi_{u}\right]$ & I & Unsigned, flux-weighted conv./div. flows & $"$ \\
\hline$\sum(\nabla \cdot \mathbf{u})$ & $\mathrm{E}$ & conv./div. flows & $"$ \\
\hline $\bar{\sum}|\nabla \cdot \mathbf{u}|$ & $\mathrm{E}$ & Unsigned conv./div. flows & $"$ \\
\hline$\sum|d \Phi|(\nabla \cdot \mathbf{u})$ & $\mathrm{E}$ & Flux-weighted, signed conv./div. flows & $"$ \\
\hline$\sum|d \Phi(\nabla \cdot \mathbf{u})|$ & $\mathrm{E}$ & Unsigned, flux-weighted conv./div. flows & $"$ \\
\hline$D_{\mathrm{COF}}$ & $\mathrm{E}$ & Convergence of AR flux & Wang 2006 \\
\hline$S_{\mathrm{COF}}$ & $\mathrm{E}$ & Shearing of AR flux & $"$ \\
\hline$R$ & $\mathrm{E}$ & Flux near PILs & $\begin{array}{l}\text { Falconer et al. 2003; } \\
\text { Schrijver } 2007\end{array}$ \\
\hline$\dot{R}$ & $\mathrm{E}$ & Change in flux near PILs & $"$ \\
\hline$\dot{R} / R$ & $\mathrm{I}$ & Change in flux near PILs & $"$ \\
\hline
\end{tabular}


Table 2-Continued

\begin{tabular}{|c|c|c|c|}
\hline Property & Scaling $^{\mathrm{a}}$ & Related Physical Quantity & Citation $(\mathrm{s})^{\mathrm{b}}$ \\
\hline$S_{R}=\sum u \tilde{B}_{R}^{2}$ & $\mathrm{E}$ & Poynting flux proxy & - \\
\hline $\mathcal{M}\left[u_{c}\right]$ & $\mathrm{I}$ & Flows along contours of $\tilde{B}_{R}$ & Démoulin \& Berger 2003 \\
\hline $\mathcal{M}\left[u_{c}|d \Phi| / \Phi_{u}\right]$ & $\mathrm{I}$ & Flux-weighted contour flows & $"$ \\
\hline $\mathcal{M}\left[u_{g}\right]$ & I & Flows along gradients of $\tilde{B}_{R}$ & - \\
\hline $\mathcal{M}\left[u_{g}|d \Phi| / \Phi_{u}\right]$ & I & Flux-weighted gradient flows & - \\
\hline $\mathcal{M}\left[W u_{g} \tilde{B}_{r}\right]$ & I & Converging motions along PIL & Linker et al. 2003 \\
\hline$\sum W u_{g} \tilde{B}_{r}$ & $\mathrm{E}$ & $"$ & $"$ \\
\hline$\overline{\mathcal{M}}\left[W u_{c} \tilde{B}_{r}\right]$ & $\mathrm{I}$ & Shearing motions along PIL & Antiochos et al. 1999 \\
\hline$\sum W u_{c} \tilde{B}_{r}$ & $\mathrm{E}$ & $"$ & $"$ \\
\hline$\sum \tilde{B}_{R}^{2}$ & $\mathrm{E}$ & Component of Photospheric Magnetic Energy & - \\
\hline$\sum u^{2}$ & $\mathrm{E}$ & Kinetic energy & - \\
\hline$\sum\left|\tilde{B}_{R}\right| u^{2}$ & $\mathrm{E}$ & Field-weighted kinetic energy & - \\
\hline
\end{tabular}

${ }^{\mathrm{a}} \mathrm{I}=$ intensive, $\mathrm{E}=$ extensive

${ }^{b}$ References chosen are representative, not necessarily either exhaustive or initial.

Table 3. Magnetic Flux Correlations

\begin{tabular}{cccccc}
\hline \hline & $\Phi$ & $R$ & $\dot{\Phi}$ & $\dot{R}$ & $\left|\tilde{B}_{R}\right|^{2}$ \\
\hline$\Phi$ & - & $0.69^{\mathrm{b}}$ & $0.18^{\mathrm{b}}$ & $0.19^{\mathrm{b}}$ & $0.91^{\mathrm{b}}$ \\
$R$ & $0.53^{\mathrm{a}}$ & - & $0.60^{\mathrm{b}}$ & $0.32^{\mathrm{b}}$ & $0.84^{\mathrm{b}}$ \\
$\dot{\Phi}$ & $0.24^{\mathrm{a}}$ & $0.54^{\mathrm{a}}$ & - & $0.48^{\mathrm{b}}$ & $0.40^{\mathrm{b}}$ \\
$\dot{R}$ & $0.21^{\mathrm{a}}$ & $0.54^{\mathrm{a}}$ & $0.48^{\mathrm{a}}$ & - & $0.17^{\mathrm{b}}$ \\
$\left|\tilde{B}_{R}\right|^{2}$ & $0.88^{\mathrm{a}}$ & $0.61^{\mathrm{a}}$ & $0.50^{\mathrm{a}}$ & $0.23^{\mathrm{a}}$ & - \\
\hline
\end{tabular}

a linear (Pearson) correlation

${ }^{\mathrm{b}}$ rank-order (Spearman) correlation 
Table 4. Flare Frequencies and Skill Scores

\begin{tabular}{|c|c|c|c|c|c|c|c|}
\hline Wind. & Code & Variable(s) Considered [Disc. Coeff.] & $\mathrm{PF} / \mathrm{F}^{\mathrm{a}}$ & $\mathrm{PNF} / \mathrm{F}$ & $\mathrm{PF} / \mathrm{NF}$ & $\mathrm{PNF} / \mathrm{NF}$ & $S S$ \\
\hline \multirow[t]{3}{*}{$6 \mathrm{C}$} & DAVE & $R$ & 79 & 237 & 47 & 2345 & 0.16 \\
\hline & & $R[1.24], S_{R}[1.12]$ & 102 & 214 & 67 & 2325 & 0.19 \\
\hline & & $R[1.19], S_{R}[0.77],\left\langle\left|\tilde{B}_{R}\right|\right\rangle[0.52]$ & 106 & 210 & 70 & 2322 & 0.20 \\
\hline \multirow[t]{3}{*}{$6 \mathrm{C}$} & FLCT & $S_{R}$ & 103 & 213 & 76 & 2316 & 0.16 \\
\hline & & $S_{R}[1.21], R[1.16]$ & 107 & 209 & 70 & 2322 & 0.20 \\
\hline & & $S_{R}[1.64], R[1.11], \sum\left|\tilde{B}_{R}\right| u^{2}[-0.47]$ & 107 & 209 & 68 & 2324 & 0.20 \\
\hline \multirow[t]{3}{*}{$6 \mathrm{~N}$} & DAVE & $R$ & 39 & 80 & 62 & 2527 & -0.08 \\
\hline & & $R[2.01], S_{R}[0.76]$ & 40 & 79 & 72 & 2517 & -0.08 \\
\hline & & $R[1.98], S_{R}[0.60],\left\langle\left|\tilde{B}_{R}\right|\right\rangle[0.44]$ & 42 & 77 & 67 & 2522 & -0.05 \\
\hline \multirow[t]{3}{*}{$6 \mathrm{~N}$} & FLCT & $R$ & 39 & 80 & 62 & 2527 & -0.08 \\
\hline & & $R[2.01], S_{R}[0.71]$ & 41 & 78 & 66 & 2523 & -0.07 \\
\hline & & $R[1.99], S_{R}[0.53], \Phi[0.47]$ & 42 & 77 & 64 & 2525 & -0.04 \\
\hline \multirow[t]{3}{*}{$24 \mathrm{C}$} & DAVE & $S_{R}$ & 290 & 383 & 68 & 1967 & 0.33 \\
\hline & & $S_{R}[1.19],\left\langle\left|\tilde{B}_{R}\right|\right\rangle[1.08]$ & 348 & 325 & 101 & 1934 & 0.37 \\
\hline & & $S_{R}[1.63],\left\langle\left|\tilde{B}_{R}\right|\right\rangle[0.95], \sum\left|\tilde{B}_{R}\right| u^{2}[-0.39]$ & 349 & 324 & 90 & 1945 & 0.37 \\
\hline \multirow[t]{3}{*}{$24 \mathrm{C}$} & FLCT & $S_{R}$ & 299 & 374 & 72 & 1963 & 0.33 \\
\hline & & $S_{R}[1.22],\left\langle\left|\tilde{B}_{R}\right|\right\rangle[1.03]$ & 335 & 338 & 76 & 1959 & 0.37 \\
\hline & & $S_{R}[1.19],\left\langle\left|\tilde{B}_{R}\right|\right\rangle[0.71], \sigma\left[\tilde{B}_{R} \mid\right][0.37]$ & 330 & 343 & 76 & 1959 & 0.37 \\
\hline \multirow[t]{3}{*}{$24 \mathrm{~N}$} & DAVE & $S_{R}$ & 107 & 221 & 83 & 2297 & 0.15 \\
\hline & & $S_{R}[1.19], R[0.85]$ & 106 & 222 & 72 & 2308 & 0.17 \\
\hline & & $S_{R}[0.82], R[0.78],\left\langle\left|\tilde{B}_{R}\right|\right\rangle[0.55]$ & 105 & 223 & 73 & 2307 & 0.18 \\
\hline \multirow[t]{3}{*}{$24 \mathrm{~N}$} & FLCT & $S_{R}$ & 107 & 221 & 74 & 2306 & 0.16 \\
\hline & & $S_{R}[1.34], R[0.74]$ & 112 & 216 & 67 & 2313 & 0.18 \\
\hline & & $S_{R}[1.35], R[0.47], \mathcal{F}_{24, \text { current }}[0.45]$ & 115 & 213 & 63 & 2317 & 0.20 \\
\hline
\end{tabular}

${ }^{\mathrm{a}} \mathrm{PF}=$ predicted to flare $\mathrm{F}=$ flare(s) occurred; $\mathrm{PNF}=$ predicted no flare; $\mathrm{NF}=$ no flare occurred 
Table 5. Means of Selected Raw and Standardized Variables for Flaring and Non-Flaring Populations

\begin{tabular}{|c|c|c|c|c|c|c|c|}
\hline Window & Variable [Units] ${ }^{\mathrm{a}}$ & $\overline{\mathrm{F}}^{\mathrm{b}}$, raw & $\overline{\mathrm{NF}}^{\mathrm{C}}$, raw & $\overline{\mathrm{F}+\mathrm{NF}}$, raw & Std. Dev. ${ }^{\mathrm{d}}$ & $\overline{\mathrm{F}}$, standardized $^{\mathrm{e}}$ & $\overline{\mathrm{NF}}$, standardized \\
\hline \multirow[t]{2}{*}{$6 \mathrm{C}$} & $S_{R}\left[\mathrm{~km} \mathrm{G}^{2} / \mathrm{s}\right]$ & $3.6 \times 10^{7}$ & $1.4 \times 10^{7}$ & $1.6 \times 10^{7}$ & $1.6 \times 10^{7}$ & 1.3 & $-1.7 \times 10^{-1}$ \\
\hline & $R[\mathrm{G}]$ & $7.3 \times 10^{3}$ & $1.0 \times 10^{3}$ & $1.8 \times 10^{3}$ & $4.5 \times 10^{3}$ & 1.2 & $-1.6 \times 10^{-1}$ \\
\hline \multirow[t]{2}{*}{$6 \mathrm{~N}$} & $R[\mathrm{G}]$ & $1.0 \times 10^{4}$ & $1.4 \times 10^{3}$ & $1.8 \times 10^{3}$ & $4.5 \times 10^{3}$ & 1.9 & $-8.8 \times 10^{-2}$ \\
\hline & $S_{R}\left[\mathrm{~km} \mathrm{G}^{2} / \mathrm{s}\right]$ & $4.0 \times 10^{7}$ & $1.5 \times 10^{7}$ & $1.6 \times 10^{7}$ & $1.6 \times 10^{7}$ & 1.5 & $-7.0 \times 10^{-2}$ \\
\hline \multirow[t]{2}{*}{$24 \mathrm{C}$} & $S_{R}\left[\mathrm{~km} \mathrm{G}^{2} / \mathrm{s}\right]$ & $3.2 \times 10^{7}$ & $1.1 \times 10^{7}$ & $1.6 \times 10^{7}$ & $1.6 \times 10^{7}$ & 0.99 & $-3.3 \times 10^{-1}$ \\
\hline & $\left\langle\left|\tilde{B}_{R}\right|\right\rangle[\mathrm{G}]$ & $5.1 \times 10^{1}$ & $3.1 \times 10^{1}$ & $3.6 \times 10^{1}$ & $1.6 \times 10^{1}$ & 0.97 & $-3.2 \times 10^{-1}$ \\
\hline \multirow[t]{2}{*}{$24 \mathrm{~N}$} & $S_{R}\left[\mathrm{~km} \mathrm{G}^{2} / \mathrm{s}\right]$ & $3.6 \times 10^{7}$ & $1.4 \times 10^{7}$ & $1.6 \times 10^{7}$ & $1.6 \times 10^{7}$ & 1.2 & $-1.7 \times 10^{-1}$ \\
\hline & $R[\mathrm{G}]$ & $6.5 \times 10^{3}$ & $1.1 \times 10^{3}$ & $1.8 \times 10^{3}$ & $4.5 \times 10^{3}$ & 1.1 & $-1.5 \times 10^{-1}$ \\
\hline
\end{tabular}

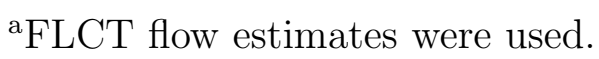

${ }^{\mathrm{b}}$ Overlines denote averages; $\mathrm{F}=$ flare $(\mathrm{s})$ occurred

${ }^{\mathrm{c}} \mathrm{NF}=$ no flare occurred

dStandard deviation of combined F+NF populations.

e Derived by subtracting the mean from raw data, then dividing by the standard deviation. 


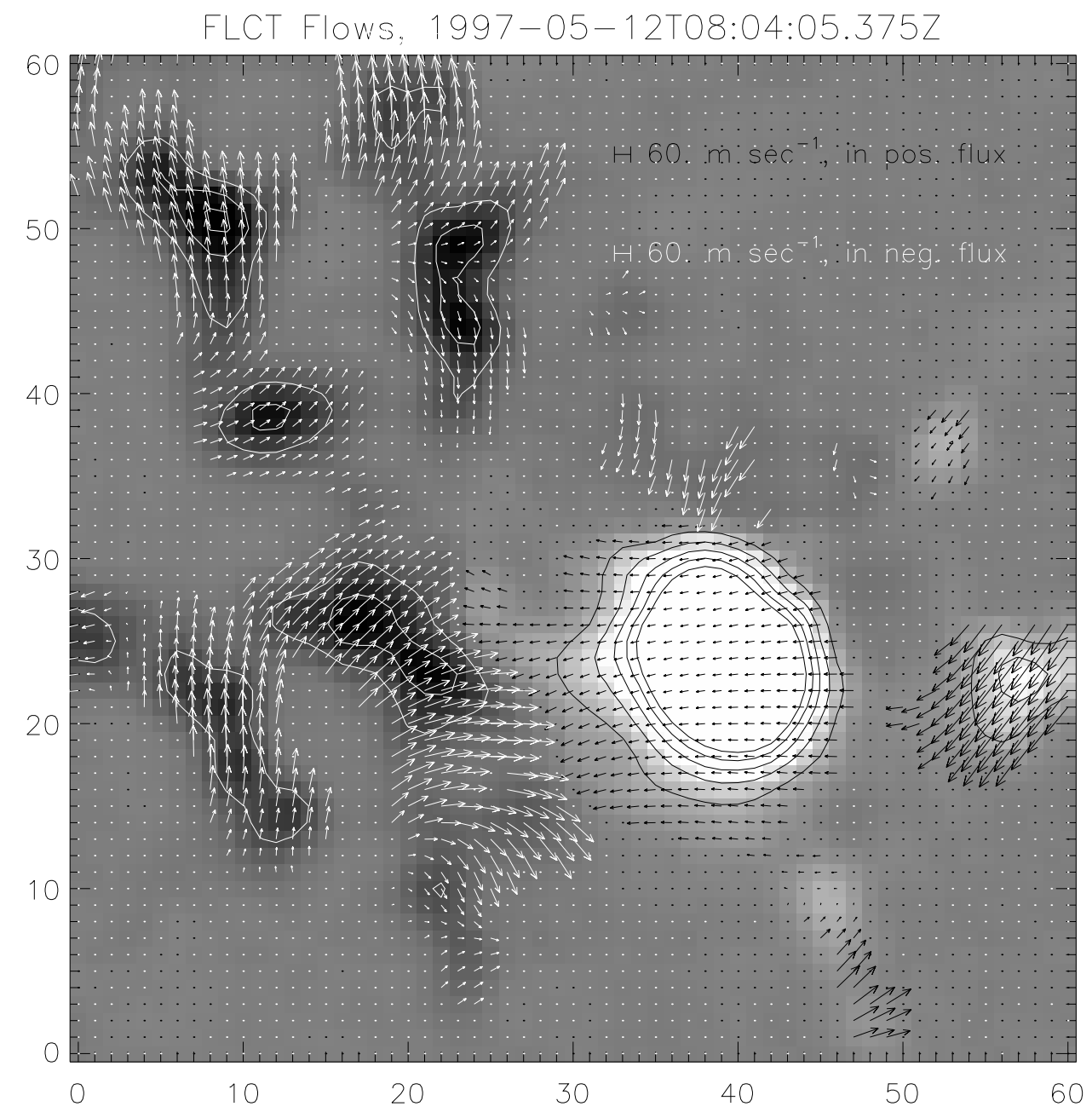

Fig. 1. - FLCT flows overlaid on a grayscale image of $\bar{B}_{R}$ from AR 8038; white and black vectors represent flows in negative and positive regions, respectively. White and black contours of $\bar{B}_{R}$ are overplotted in negative and positive regions, respectively, at $100 \mathrm{G}$ intervals between $100 \mathrm{G}$ and $500 \mathrm{G}$. The time shown at top corresponds to the end of the 96-min. interval over which flows were estimated. 


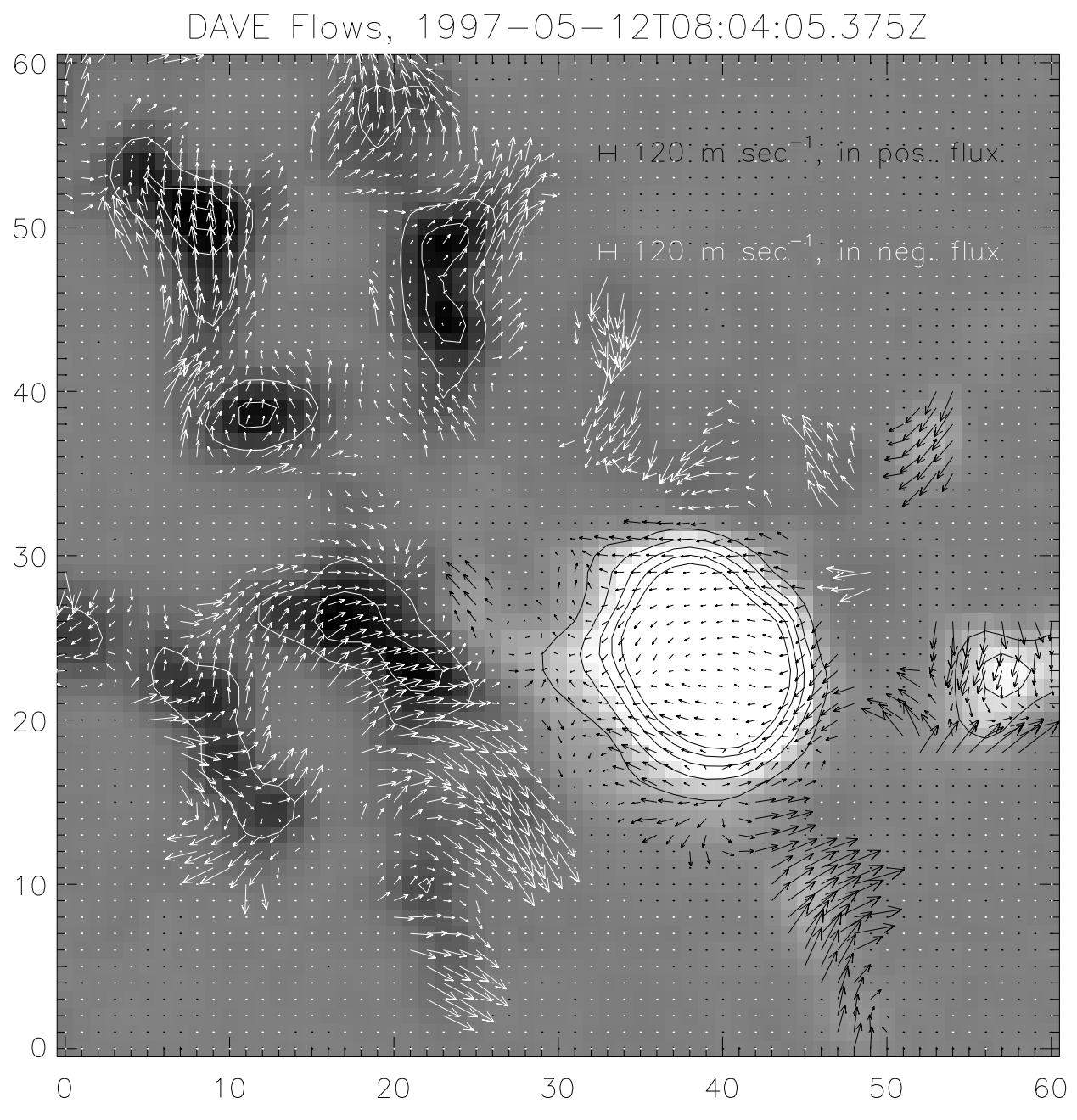

Fig. 2.- DAVE flows overlaid on a grayscale image of $\bar{B}_{R}$ from AR 8038; white and black vectors represent flows in negative and positive regions, respectively. White and black contours of $\bar{B}_{R}$ are overplotted in negative and positive regions, respectively, at $100 \mathrm{G}$ intervals between $100 \mathrm{G}$ and $500 \mathrm{G}$. The time shown at top corresponds to the end of the 96-min. interval over which flows were estimated. Note that the vector length scale differs from that in Figure 1. 

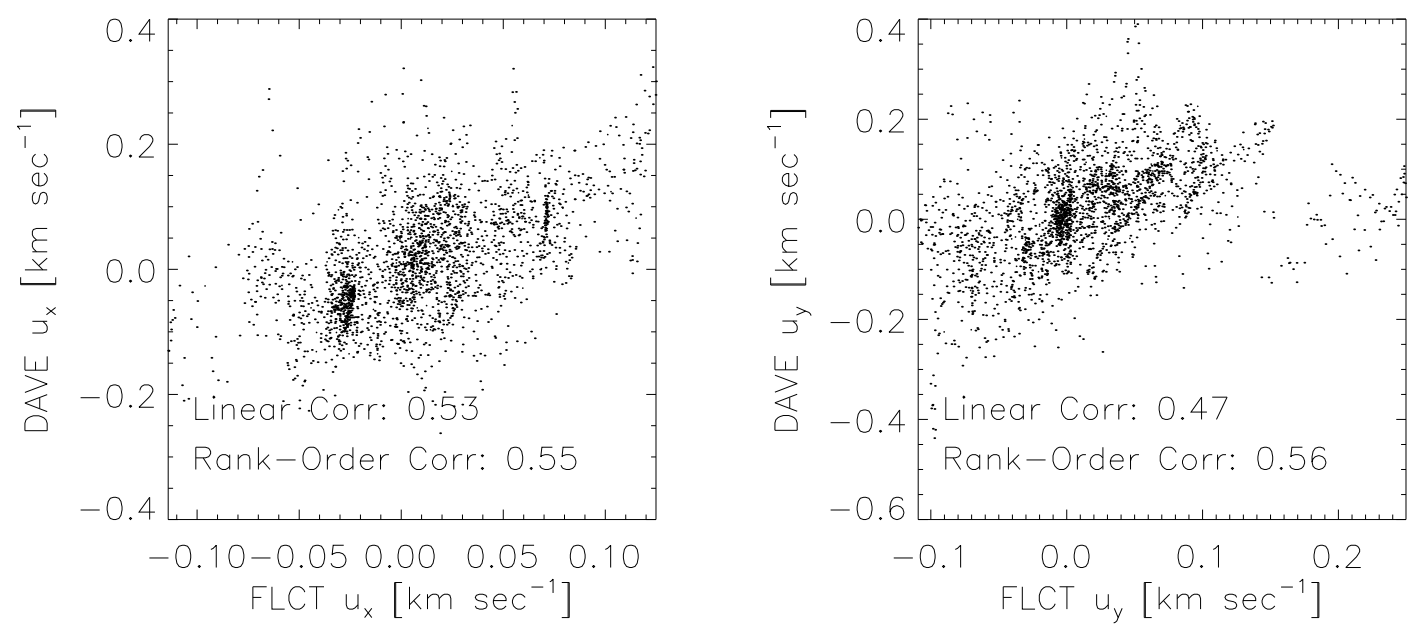

Fig. 3.- FLCT and DAVE flows from Figures 1 and 2, respectively, are compared in scatter plots of $u_{x}$ (left) and $u_{y}$ (right). We also show linear and rank-order correlation coefficients between the flow components. 

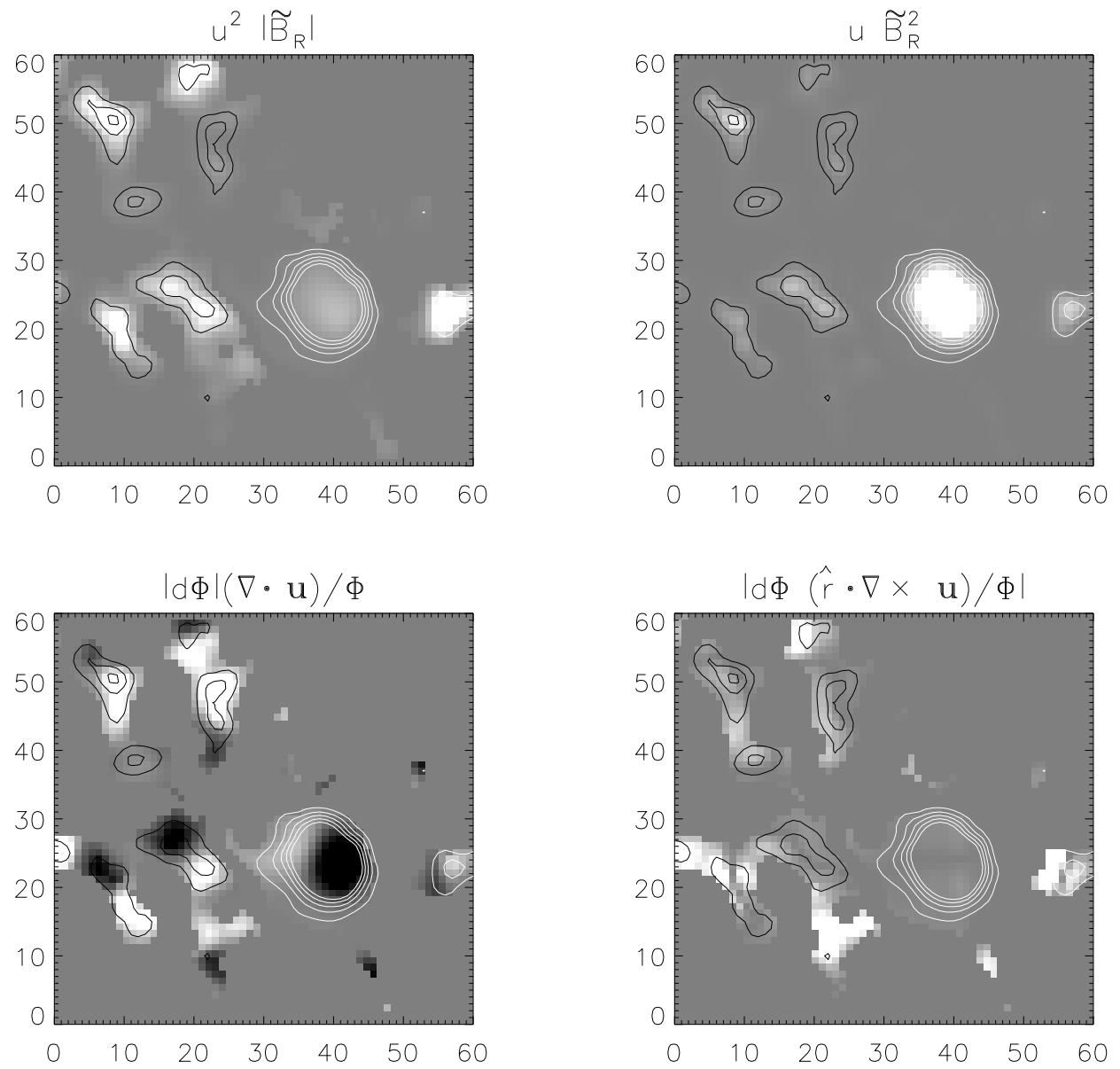

Fig. 4. - Examples of flow properties from FLCT flows corresponding to the flow field shown in Figure 1. Top row: Grayscale maps of $u^{2}\left|\bar{B}_{R}\right|$ (left) $u \bar{B}_{R}^{2}$ (right). Black and white contours of $\bar{B}_{R}$ are overplotted in negative and positive regions, respectively, at $100 \mathrm{G}$ intervals between $100 \mathrm{G}$ and $500 \mathrm{G}$, for all panels. Bottom row: Grayscale maps of $|d \Phi|$-weighted versions of the horizontal divergence (left) and unsigned vertical curl (right). 


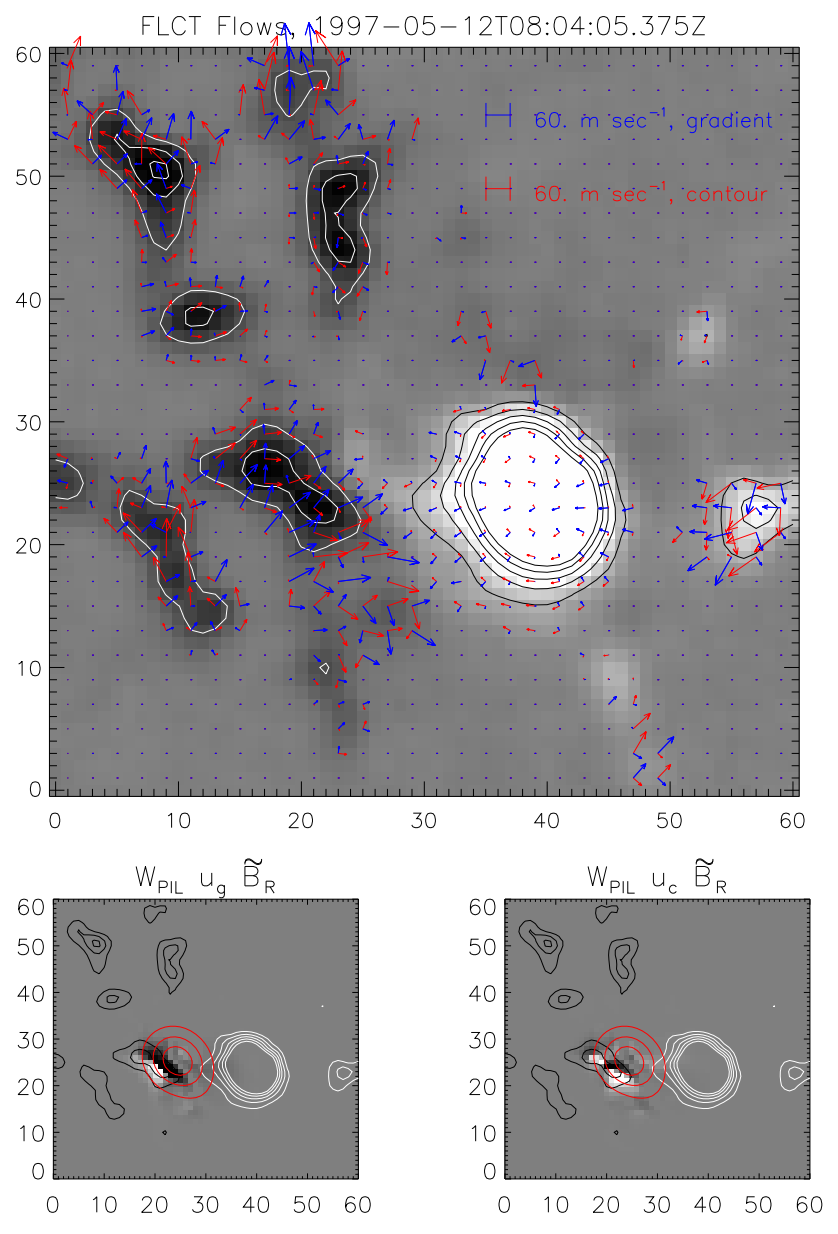

Fig. 5.- Top: The background grayscale shows $\bar{B}_{R}$ (white is positive, black negative), and white and black contours of $\bar{B}_{R}$ are overplotted in negative and positive regions, respectively, at $100 \mathrm{G}$ intervals between $100 \mathrm{G}$ and $500 \mathrm{G}$. Components of FLCT flows from Fig. 1 that lie along gradients and contours of $\bar{B}_{R}$ are shown with blue and red vectors, respectively. For clarity, not every vector is plotted. Bottom left: A grayscale map of $W_{P I L^{-}}$and $\bar{B}_{R^{-}}$weighted gradient flows, used to quantify converging motions. Black and white contours of $\bar{B}_{R}$ are overplotted in negative and positive regions, respectively, at $100 \mathrm{G}$ intervals between $100 \mathrm{G}$ and $500 \mathrm{G}$. Red contours of the PIL-weighting map $W_{P I L}$ are overlaid. Bottom right: A grayscale map of $W_{P I L^{-}}$ and $\bar{B}_{R}$-weighted contour flows, used to quantify shearing motions. All other image components are as with the bottom left image. 

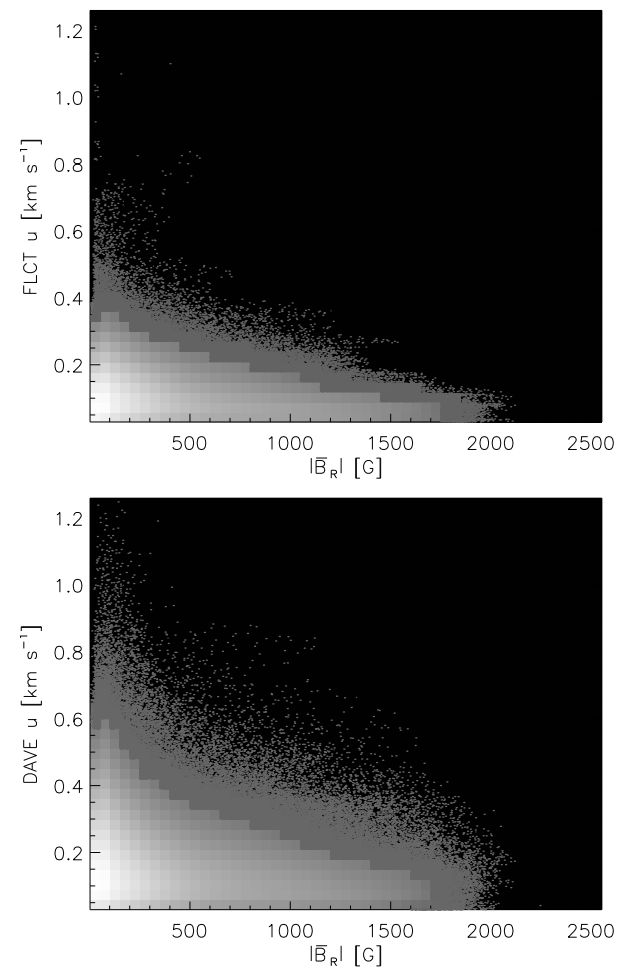

Fig. 6. - Combined log-scaled shading histogram and scatter plot of speed estimates from FLCT (top) and DAVE (bottom) as a function of pixel-averaged field strength $\bar{B}_{R}$ (see text). The grayscale for FLCT ranges from $2.4-6.2$, and for DAVE from 2.4 - 6.0. Higher speeds tend to be seen in weaker fields. 


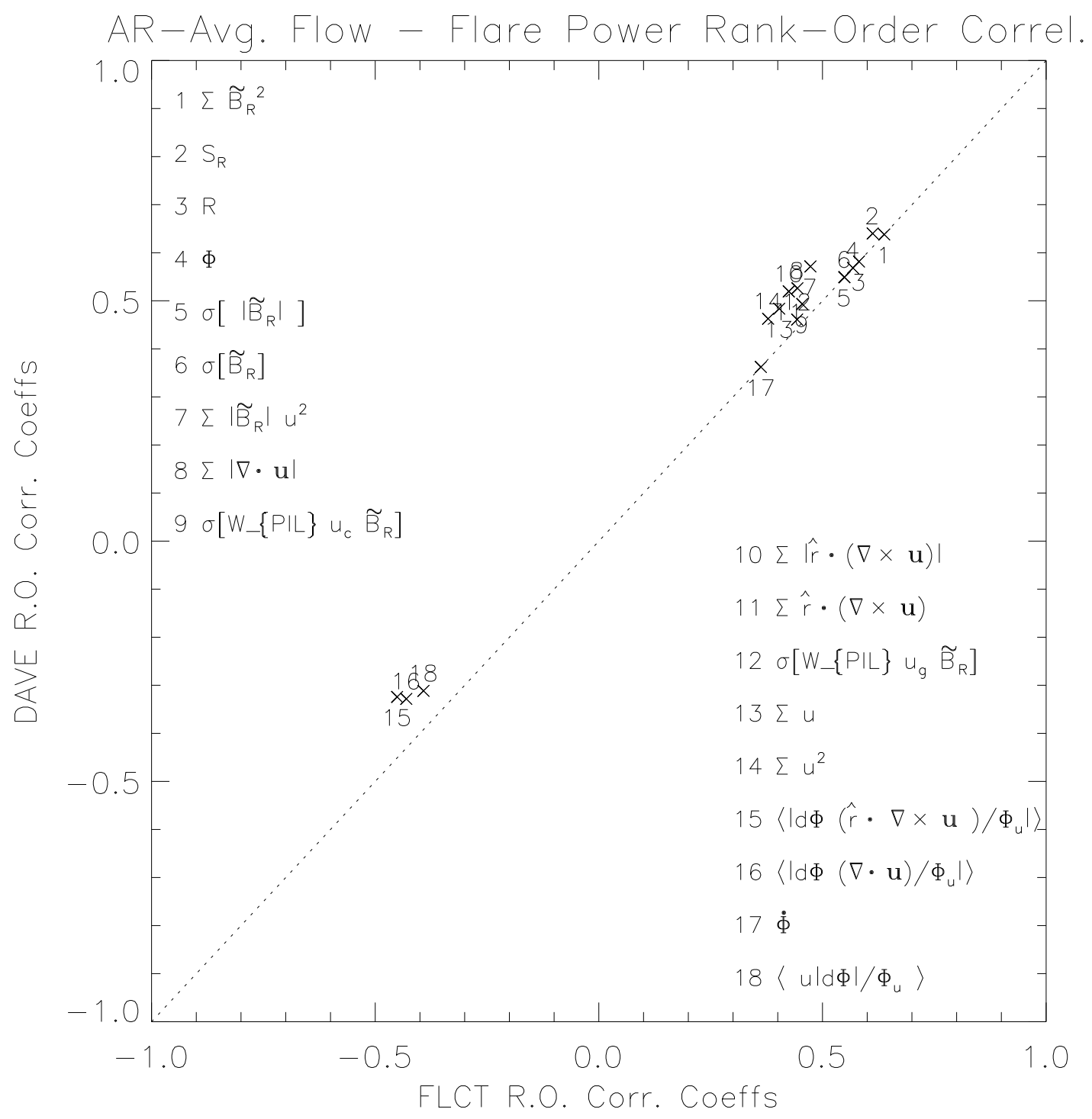

Fig. 7.- FLCT and DAVE rank-order correlation coefficients between whole-diskpassage averaged flow properties and flare power, averaged over the 46 active regions in our study. To the extent that correlation coefficients lie on the slope $=1$ dashed line (not a fit), the averaged flow properties from the FLCT and DAVE methods agree. The quantities correlated are numbered by distance from $(0,0)$, farthest first; hence, smaller numerical labels imply stronger correlation. Many of the strongest correlations do not require estimating flows. Even ranks are plotted above their symbols, and odd ranks below. 


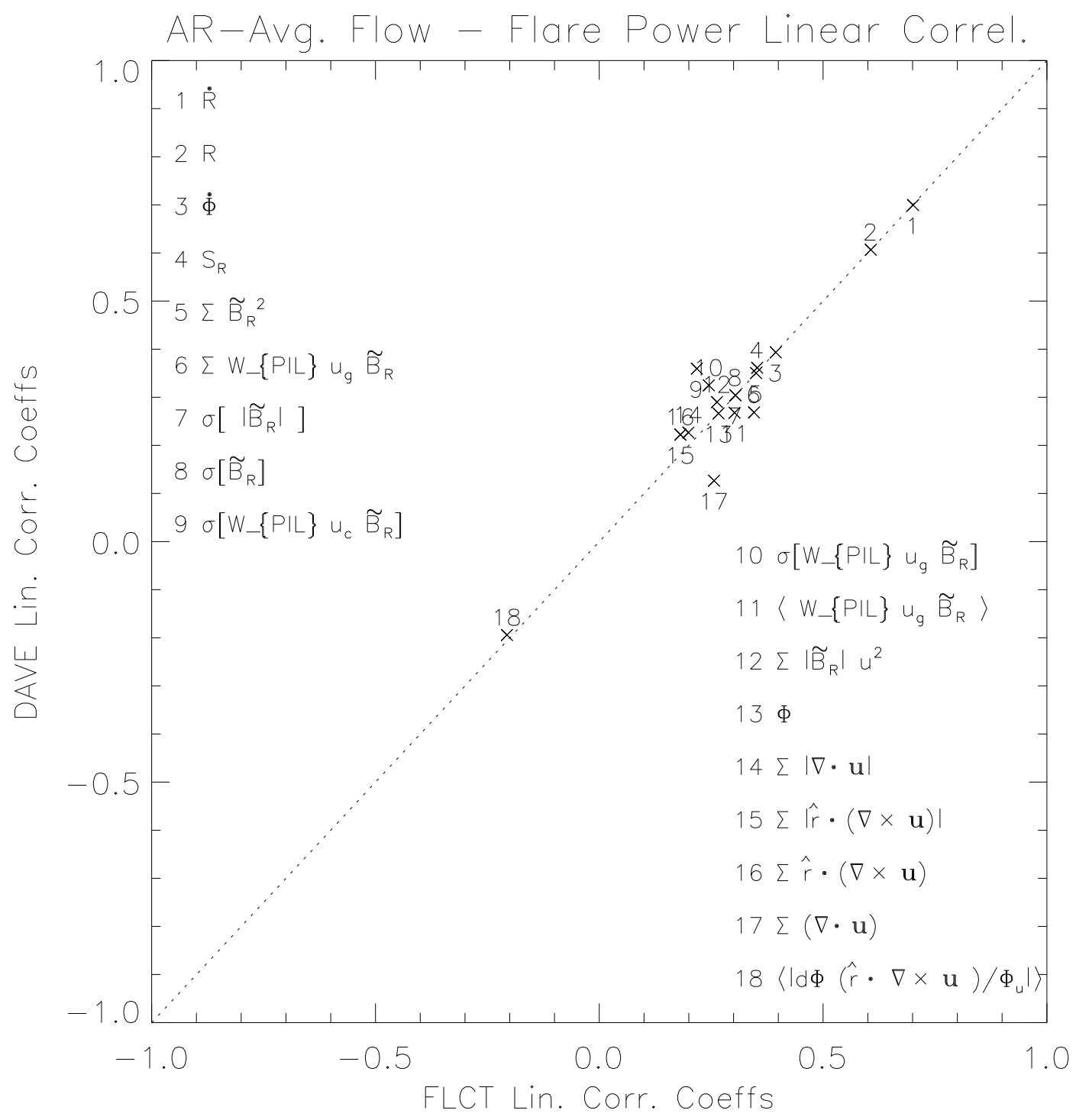

Fig. 8.- FLCT and DAVE linear correlation coefficients between whole-disk-passage averaged flow properties and flare power, averaged over the 46 active regions in our study. To the extent that correlation coefficients lie on the slope $=1$ dashed line $(n o t$ a fit), the averaged flow properties from FLCT and DAVE methods agree. The correlations are numbered by distance from $(0,0)$, farthest first; hence, smaller numerical labels imply stronger correlation. As in rank-order correlations in Figure 8, many of the strongest correlations do not require estimating flows. Even ranks are plotted above their symbols, and odd ranks below. 
AR 7981, 1996-08-01 to 1996-08-04
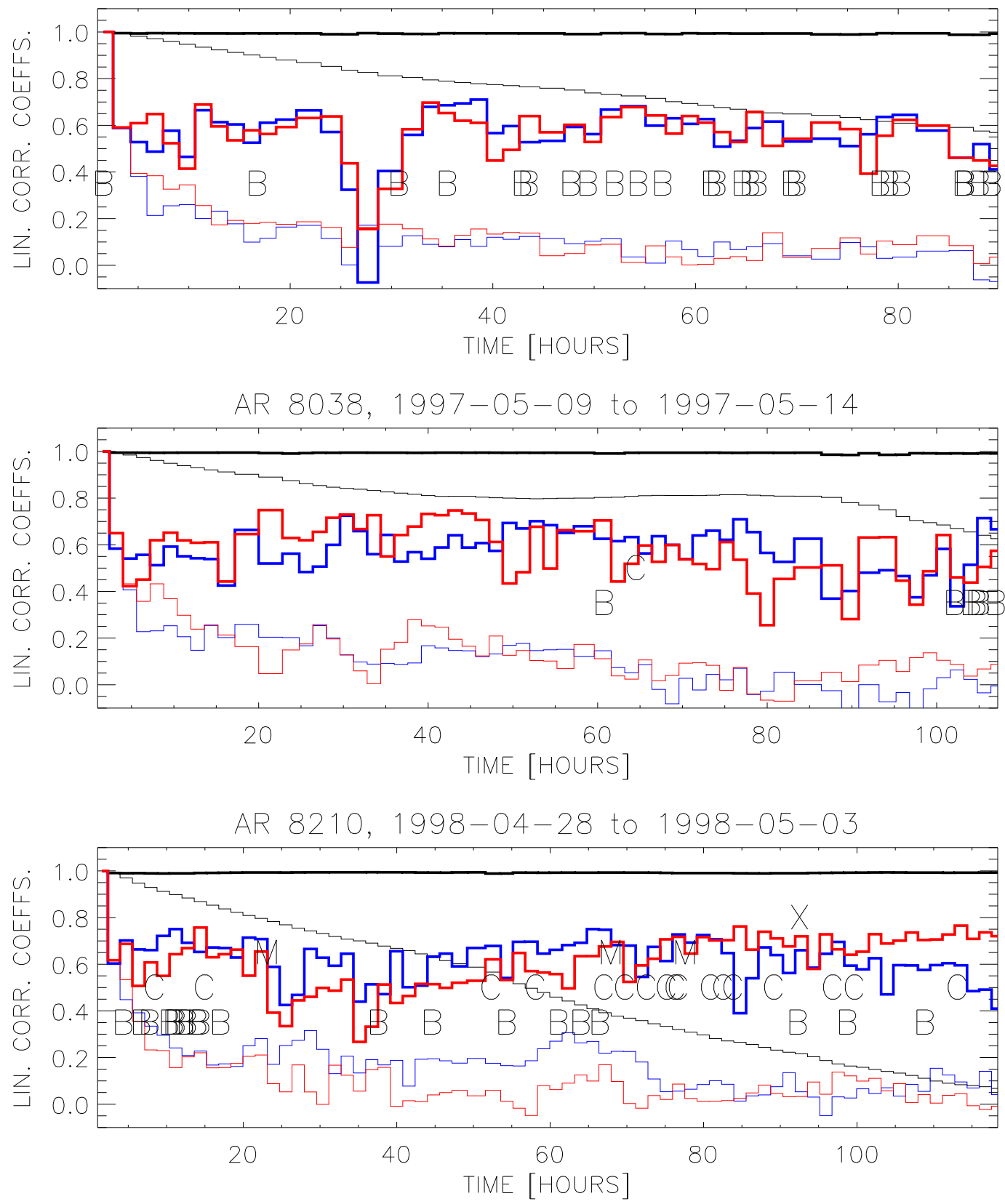

Fig. 9.- For ARs 7981,8038 , and 8210 , frame-to-frame correlations in $\bar{B}_{R}$ with time are shown in black; the thick lines represent linear correlation coefficients between the current and previous frames, while the thin lines show linear correlation coefficients between the current and initial frames. Analogous correlations are also shown for FLCT's estimated $u_{x} \& u_{y}$ in blue \& red (resp.), with thick \& thin lines denoting current-previous \& current-initial correlations (resp.). In addition, flare start times are shown by letters representing GOES flare class; the vertical placements of letters do not scale with actual flare fluxes. 

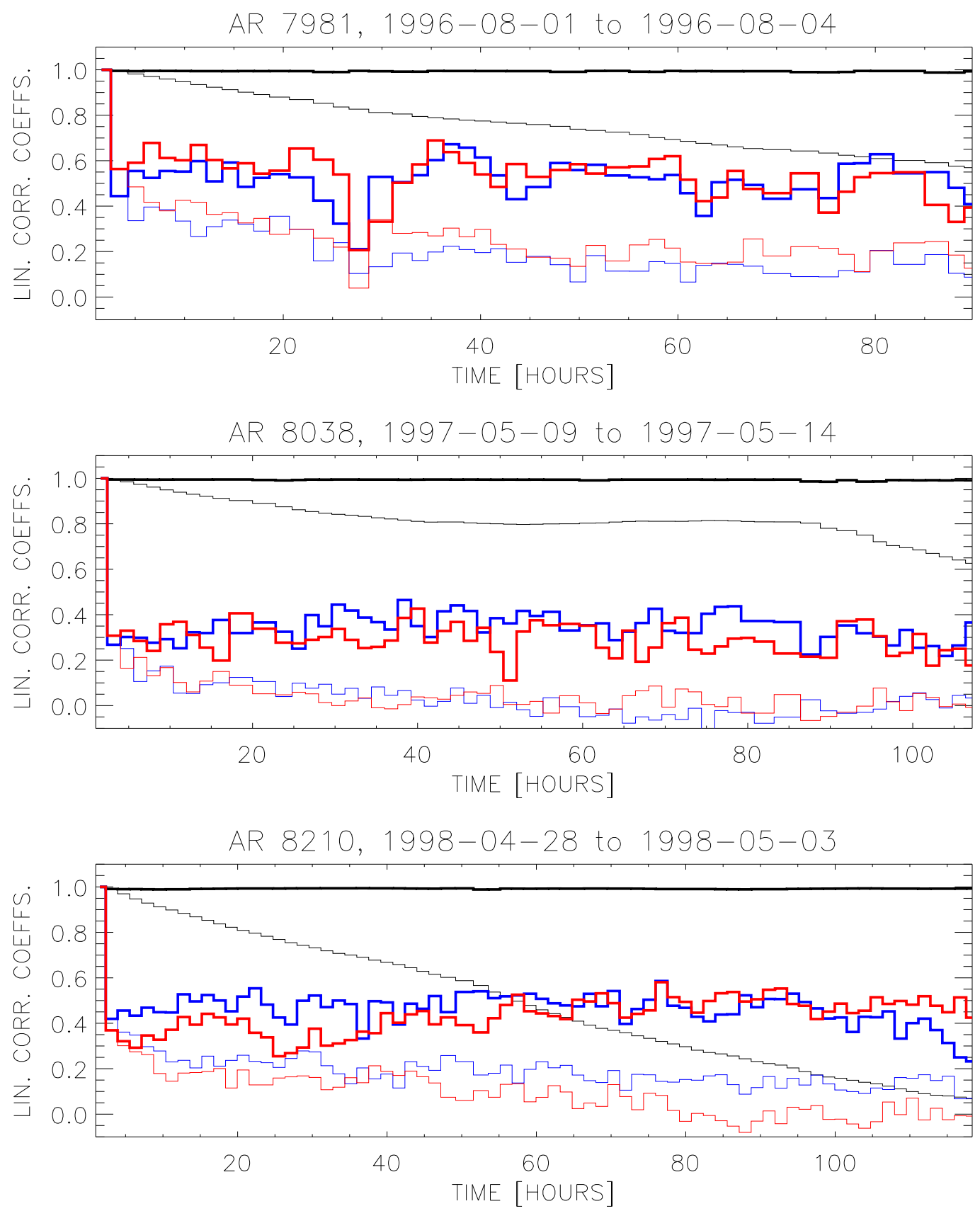

Fig. 10.- As with Figure 9, but with correlations using DAVE's $u_{x} \& u_{y}$ in blue \& red, resp. 

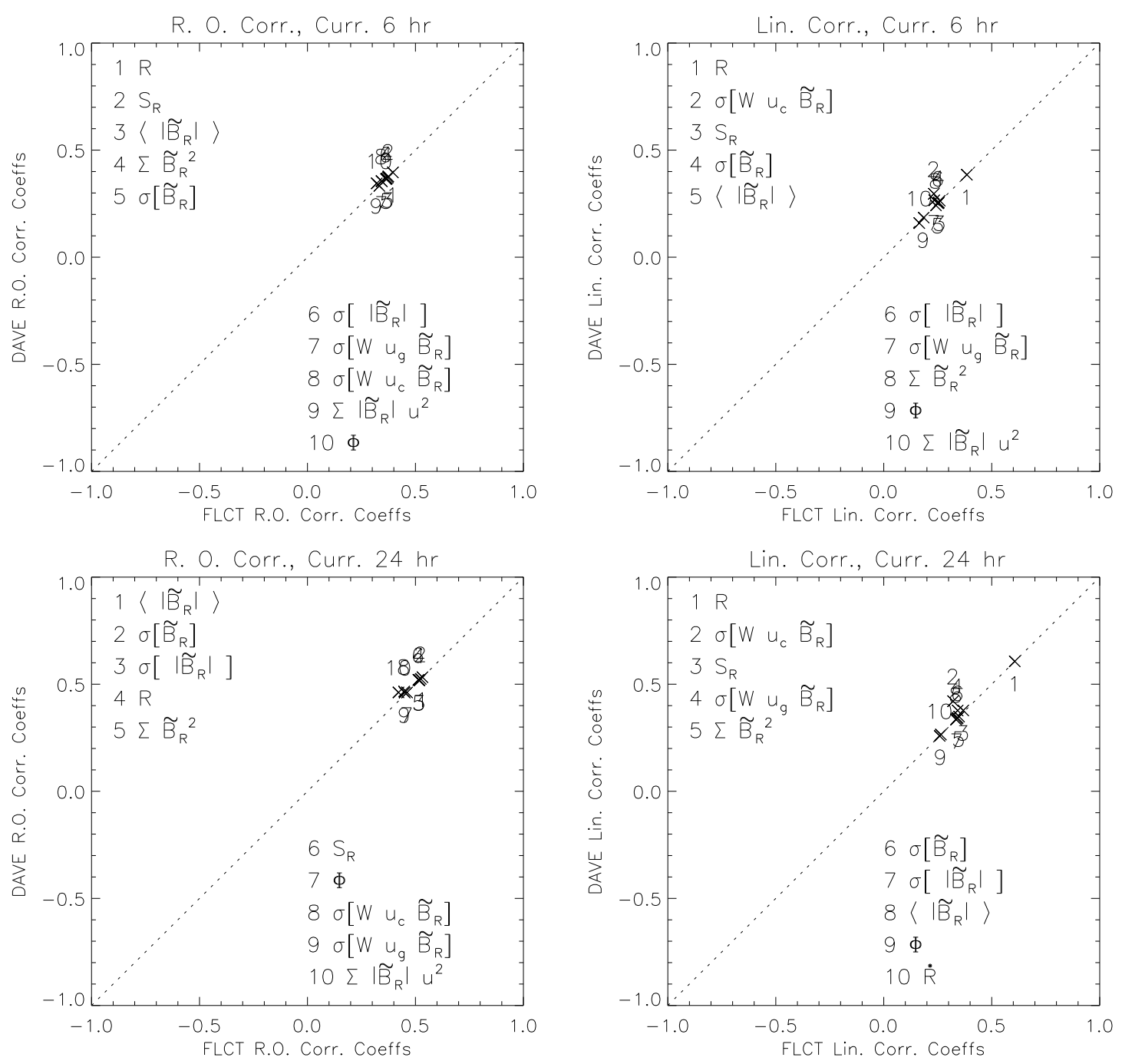

Fig. 11. - Top row: Scatter plots of rank-order (R.O.) and linear correlation coefficients (left and right panels, resp.) between average flare power and magnetogram or flow properties from FLCT (horizontal axis) and DAVE (vertical axis), within the 6-hour time window, 6C, centered on the average time of the magnetograms used to estimate flows. Quantities are labeled in order of decreasing distance from $(0,0)$, the point on the plot which corresponds to complete lack of correlation with average flare power. Low-ranked properties are not shown. Odd rank numbers appear below their corresponding plot symbols, while even rank numbers appear above their symbols. To the extent that flare correlations with flow properties from FLCT and DAVE flow agree, they lie along the dotted line (not a fit). Quantities that do not require a flow estimate (e.g., total flux, $\Phi$ ) lie on the diagonal line. Bottom row: same as for top, but for the 24-hour time window $24 \mathrm{C}$. Some of the quantities most strongly correlated with average flare power - e.g., $R$ - do not require estimating flows. Our proxy for the Poynting flux, $S_{R}$, exhibits comparable correlations. 

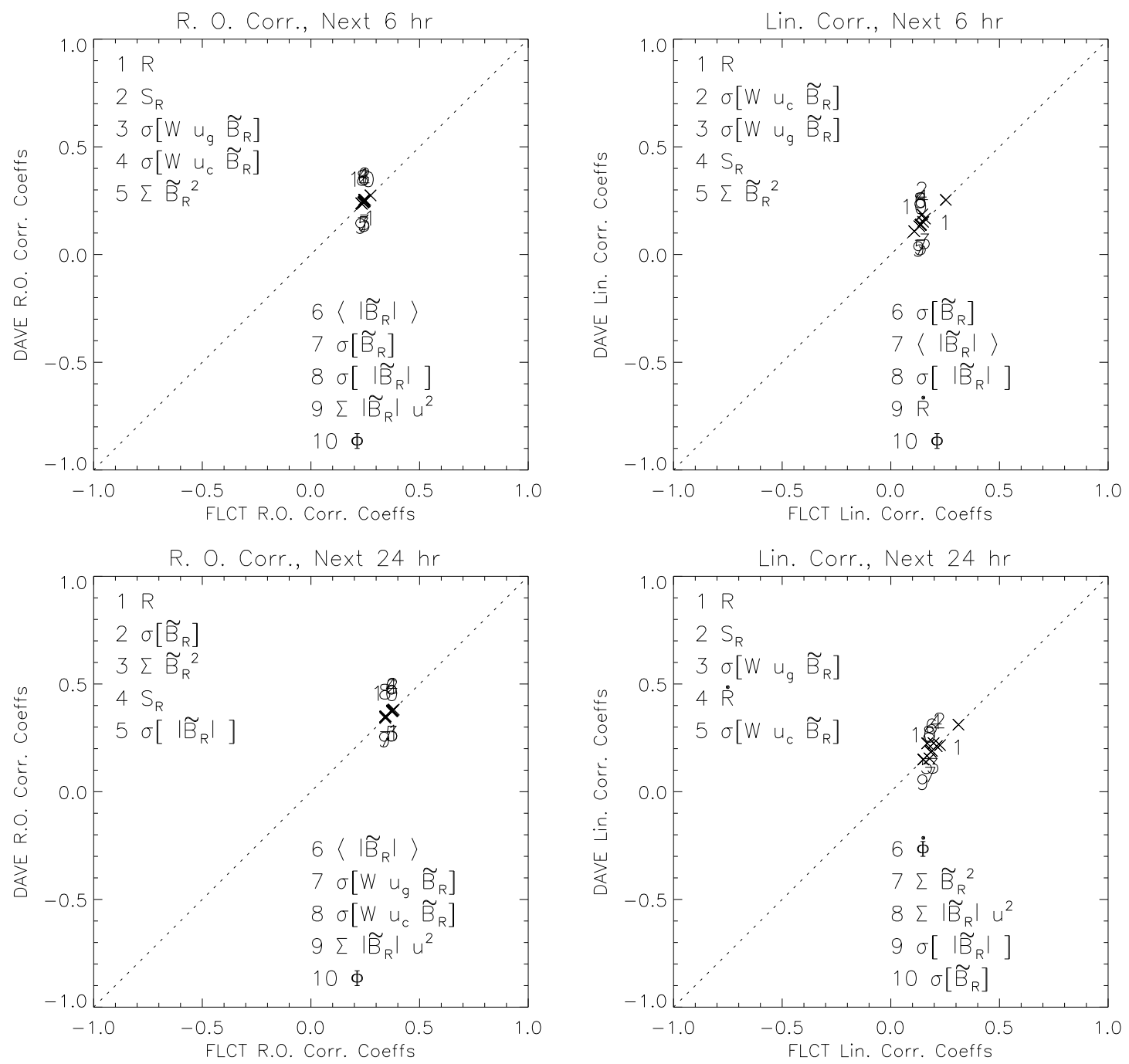

Fig. 12.- As with Figure 11, these plots show correlation coefficients between properties of magnetograms or flows from FLCT (horizontal axis) and DAVE (vertical axis), and average flare power from the $6 \mathrm{~N}$ (top row) and $24 \mathrm{~N}$ (bottom row) windows. In each panel, quantities are labeled in order of their distance from $(0,0)$. Low-ranked properties are not shown. Odd rank numbers appear below their corresponding plot symbols, while even rank numbers appear above their symbols. As with Figure 11, many quantities strongly correlated with average flare flux do not require estimating flows, but our proxy for the Poynting flux, $S_{R}$, exhibits comparable correlations. 


\section{REFERENCES}

Abbett, W. P. \& Fisher, G. H. 2003, ApJ, 582, 475

Abbett, W. P., Fisher, G. H., \& Fan, Y. 2000, ApJ, 540, 548

Abramenko, V. I. 2005, ApJ, 629, 1141

Amari, T., Luciani, J. F., Aly, J. J., Mikic, Z., \& Linker, J. 2003, ApJ, 585, 1073

Andrews, M. D. 2003, Sol. Phys., 218, 261

Antiochos, S. K., DeVore, C. R., \& Klimchuk, J. A. 1999, ApJ, 510, 485

Barnes, G. \& Forecasting the Operational All-clear Participants. 2009, in AAS/Solar Physics Division Meeting, Vol. 40, AAS/Solar Physics Division Meeting, \#16.05- - +

Barnes, G. \& Leka, K. D. 2008, ApJ, 688, L107

Barnes, G., Leka, K. D., Schumer, E. A., \& Della-Rose, D. J. 2007, Space Weather, 5, 9002

Brown, D. S., Nightingale, R. W., Alexander, D., Schrijver, C. J., Metcalf, T. R., Shine, R. A., Title, A. M., \& Wolfson, C. J. 2003, Sol. Phys., 216, 79

Canfield, R. C., Hudson, H. S., \& McKenzie, D. E. 1999, Geophys. Res. Lett., 26, 627

Chae, J., Moon, Y.-J., \& Park, Y.-D. 2004, Sol. Phys., 223, 39

Crosby, N. B., Ascwander, M. J., \& Dennis, B. R. 1993, Solar Phys., 143, 275

Démoulin, P. \& Berger, M. A. 2003, Sol. Phys., 215, 203

Démoulin, P. \& Pariat, E. 2009, Advances in Space Research, 43, 1013

Deng, N., Xu, Y., Yang, G., Cao, W., Liu, C., Rimmele, T. R., Wang, H., \& Denker, C. 2006, ApJ, 644,1278

Falconer, D. A., Moore, R. L., \& Gary, G. A. 2003, Journal of Geophysical Research (Space Physics), 108,11

—. 2006, ApJ, 644, 1258

Fan, Y. 2001, ApJ, 554, L111

Fan, Y., Abbett, W. P., \& Fisher, G. H. 2003, ApJ, 582, 1206

Fisher, G. H., Longcope, D. W., Metcalf, T. R., \& Pevtsov, A. A. 1998, ApJ, 508, 885

Fisher, G. H. \& Welsch, B. T. 2008, in Astronomical Society of the Pacific Conference Series, Vol. 383, Astronomical Society of the Pacific Conference Series, ed. R. Howe, R. W. Komm, K. S. Balasubramaniam, \& G. J. D. Petrie, 373-380; also arXiv:0712.4289

Forbes, T. G. 2000, JGR, 105, 23153

Harvey, K. L. \& Zwaan, C. 1993, Sol. Phys., 148, 85

Hathaway, D. H., Beck, J. G., Han, S., \& Raymond, J. 2002, Sol. Phys., 205, 25

Hills, M. 1966, Journal of the Royal Statistical Society, Series B (Methodological), 28, 1

Huberty, C. J. \& Wisenbaker, J. M. 1992, Journal of Educational Statistics, 17, 75 


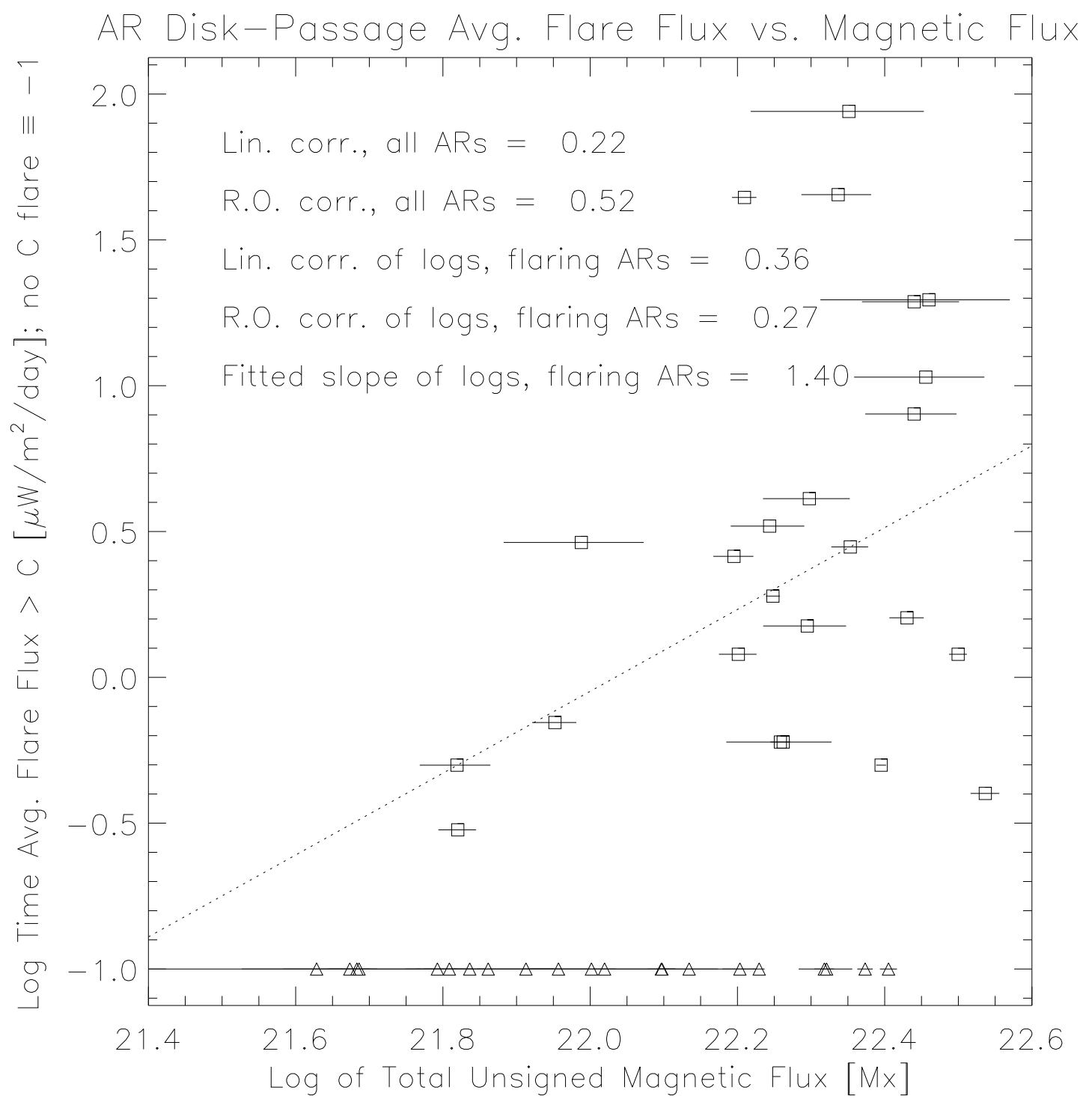

Fig. 13. - Squares show $\log _{10}$ average flux from flares above GOES C1.0 level while each AR was within $45^{\circ}$ of disk center vs. log average total unsigned flux in these ARs over that time interval. Horizontal bars show log of RMS variations flux during that interval; errors from magnetograph noise for any single flux measurement are about the size of the plotted symbols or smaller. Triangles show log of magnetic flux in the 22 ARs that did not flare above GOES C1.0 level. We also list: the linear and rank-order (R.O.) correlation coefficients between the raw magnetic and flare fluxes for all ARs; the linear and R.O. coefficients between the logs of magnetic and flare fluxes for flaring ARs; and the slope of a least- absolute- deviation fit (dotted line) of the log of flare flux as a function of log of magnetic flux for ARs that flared above the C1.0 level. 
Hudson, H. S. 1991, Sol. Phys., 133, 357

Keil, S. L., Balasubramaniam, K. S., Bernasconi, P., Smaldone, L. A., \& Cauzzi, G. 1994, in Astronomical Society of the Pacific Conference Series, Vol. 68, Solar Active Region Evolution: Comparing Models with Observations, ed. K. S. Balasubramaniam \& G. W. Simon, 265-+

Knoll, J., Leka, K. D., \& Barnes, G. 2008, LASP REU Student Presentations, unpublished, http://lasp.colorado.edu/reu/student_docs/posters/Knoll_Poster.pdf

Kucera, T. A., Dennis, B. R., Schwartz, R. A., \& Shaw, D. 1997, ApJ, 475, 338

LaBonte, B. J., Georgoulis, M. K., \& Rust, D. M. 2007, ApJ, 671, 955

Leighton, R. B., Noyes, R. W., \& Simon, G. W. 1962, ApJ, 135, 474

Leka, K. D. \& Barnes, G. 2003, ApJ, 595, 1296

—. 2007, ApJ, 656, 1173

Leka, K. D., Canfield, R. C., McClymont, A. N., \& Van Driel Gesztelyi, L. 1996, ApJ, 462, 547

Li, Y., Luhmann, J., Fisher, G., \& Welsch, B. 2004, Journal of Atmospheric and Solar-Terrestrial Physics, 66, 1271

Li, Y., Lynch, B. J., Welsch, B., Stenborg, G. A.and Luhmann, J. G., Fisher, G. H., Liu, Y., \& Nightingale, R. W. 2009, Sol. Phys., submitted,"

Linker, J. A., Lionello, R., Mikić, Z., \& Amari, T. 2001, JGR, 106, 25165

Linker, J. A., Mikić, Z., Lionello, R., Riley, P., Amari, T., \& Odstrcil, D. 2003, Physics of Plasmas, 10,1971

Lionello, R., Mikić, Z., Linker, J. A., \& Amari, T. 2002, ApJ, 581, 718

Longcope, D. W., McKenzie, D., Cirtain, J., \& Scott, J. 2005, ApJ, 630, 596

Longcope, D. W. \& Welsch, B. T. 2000, ApJ, 545, 1089

Lu, E. T. \& Hamilton, R. J. 1991, ApJ, 380, L89

Lynch, B. J., Antiochos, S. K., DeVore, C. R., Luhmann, J. G., \& Zurbuchen, T. H. 2008, ApJ, 683, 1192

Magara, T. \& Longcope, D. W. 2001, ApJ, 559, L55

Manchester, W. I. 2007, ApJ, 666, 532

McClymont, A. N. \& Fisher, G. H. 1989, in Solar System Plasma Physics, ed. J. H. Waite, J. L. Burch, \& R. L. Moore, AGU, Washington, 219

Moore, R. L., Sterling, A. C., Hudson, H. S., \& Lemen, J. R. 2001, ApJ, 552, 833

November, L. \& Simon, G. 1988, ApJ, 333, 427

Parker, E. N. 1984, ApJ, 280, 423

Pevtsov, A. A., Fisher, G. H., Acton, L. W., Longcope, D. W., Johns-Krull, C. M., Kankelborg, C. C., \& Metcalf, T. R. 2003, ApJ, 598, 1387

Priest, E. R. \& Heyvaerts, J. 1974, Sol. Phys., 36, 433 
Roussev, I. I., Sokolov, I. V., Forbes, T. G., Gombosi, T. I., Lee, M. A., \& Sakai, J. I. 2004, ApJ, $605, \mathrm{~L} 73$

Sammis, I., Tang, F., \& Zirin, H. 2000, ApJ, 540, 583

Scherrer, P., Bogart, R. S., Bush, R. I., Hoeksema, J. T., Kosovichev, A., Schou, J., Rosenberg, W., Springer, L., Tarbell, T., Title, A., Wolfson, C., Zayer, I., \& The MDI Engineering Team. 1995, Solar Phys., 162, 129

Schrijver, C. J. 2007, ApJ, 655, L117

Schrijver, C. J., DeRosa, M. L., Title, A. M., \& Metcalf, T. R. 2005, ApJ, 628, 501

Schrijver, C. J., Title, A. M., van Ballegooijen, A. A., Hagenaar, H. J., , \& Shine, R. A. 1997, ApJ, 487,424

Schuck, P. W. 2005, ApJ, 632, L53

—. 2006, ApJ, 646, 1358

-. 2008, ApJ, 683, 1134

Thompson, W. T. 2006, A\&A, 449, 791

Tokman, M. \& Bellan, P. M. 2002, ApJ, 567, 1202

Wang, H. 2006, ApJ, 649, 490

Welsch, B. T. 2006, ApJ, 638, 1101

Welsch, B. T., Abbett, W. P., DeRosa, M. L., Fisher, G. H., Georgoulis, M. K. Kusano, K., Longcope, D. W., Ravindra, B., \& Schuck, P. W. 2007, ApJ, 670, 1434

Welsch, B. T. \& Fisher, G. H. 2008, in Astronomical Society of the Pacific Conference Series, Vol. 383, Astronomical Society of the Pacific Conference Series, ed. R. Howe, R. W. Komm, K. S. Balasubramaniam, \& G. J. D. Petrie, 19-30; also arXiv:0710.0546

Welsch, B. T., Fisher, G. H., Abbett, W. P., \& Régnier, S. 2004, ApJ, 610, 1148

Welsch, B. T. \& Li, Y. 2008, in Astronomical Society of the Pacific Conference Series, Vol. 383, Astronomical Society of the Pacific Conference Series, ed. R. Howe, R. W. Komm, K. S. Balasubramaniam, \& G. J. D. Petrie, 429-437; also arXiv:0710.0562

Wheatland, M. S. 2001, Sol. Phys., 203, 87

—. 2005, Space Weather, 3, 7003

Zirin, H. \& Liggett, M. A. 1987, Sol. Phys., 113, 267

Zirin, H. \& Marquette, W. 1991, Sol. Phys., 131, 149 